Supporting Information

\title{
Synthesis and Spectral Properties of Push-Pull Dyes Based on Isobenzofuran Scaffolds
}

\author{
Sean R. Norris, ${ }^{\dagger}$ Caroline Warner, ${ }^{\dagger}$ Bryan J. Lampkin, Paige Bouc, Brett VanVeller* \\ Department of Chemistry, lowa State University, Ames, lowa 50011
}

Contents

MATERIALS AND GENERAL METHODS

S2

S2

SYNTHETIC PROCEDURES

NMR SPECTRA

S7

Figure S1: ${ }^{1 \mathrm{H}} \mathrm{NMR}$ spectra of $4(400 \mathrm{MHz}$, DMSO-d6)

S7

Figure S2: ${ }^{13} \mathrm{C}\{1 \mathrm{H}\}$ NMR spectra of $4(100 \mathrm{MHz}$, DMSO-d6)

S8

Figure S3: ${ }^{1} \mathrm{H}$ NMR spectra of $5\left(400 \mathrm{MHz}, \mathrm{CDCl}_{3}\right)$

S9

Figure S4: ${ }^{3} \mathrm{C}\{1 \mathrm{H}\} \mathrm{NMR}$ spectra of $5\left(100 \mathrm{MHz}, \mathrm{CDCl}_{3}\right)$

S10

Figure S5: ${ }^{1} \mathrm{H}$ NMR spectra of $8\left(400 \mathrm{MHz}, \mathrm{CDCl}_{3}\right)$

S11

Figure S6: ${ }^{3} \mathrm{C}\{1 \mathrm{H}\} \mathrm{NMR}$ spectra of $8\left(100 \mathrm{MHz}, \mathrm{CDCl}_{3}\right)$

S12

Figure S7: ${ }^{1} \mathrm{H}$ NMR spectra of 10-OMe $\left(400 \mathrm{MHz}^{\left.-\mathrm{CDCl}_{3}\right)}\right.$

$\mathrm{S} 13$

Figure S8: ${ }^{13} \mathrm{C}\{1 \mathrm{H}\} \mathrm{NMR}$ spectra of $10-\mathrm{OMe}\left(100 \mathrm{MHz}, \mathrm{CDCl}_{3}\right)$

S14

Figure S9: $1 \mathrm{H} \mathrm{NMR}$ spectra of $10-\mathrm{NHMe}\left(400 \mathrm{MHz}, \mathrm{CDCl}_{3}\right)$

$\mathrm{S} 15$

Figure S10: ${ }^{13} \mathrm{C}\{1 \mathrm{H}\} \mathrm{NMR}$ spectra of $10-\mathrm{NHMe}\left(100 \mathrm{MHz}, \mathrm{CDCl}_{3}\right) \quad \mathrm{S} 16$

Figure S11: ${ }^{1} \mathrm{H}$ NMR spectra of $10-\mathrm{R}^{3}(400 \mathrm{MHz}, \mathrm{DMSO}-\mathrm{d} 6) \quad \mathrm{S} 17$

Figure S12: ${ }^{13} \mathrm{C}\{1 \mathrm{H}\}$ NMR spectra of $10-\mathrm{R}^{3}(100 \mathrm{MHz}$, DMSO-d6) $\mathrm{S} 18$

Figure S13: ${ }^{1} \mathrm{H} \mathrm{NMR}$ spectra of 2-OMe $\left(400 \mathrm{MHz}, \mathrm{CDCl}_{3}\right) \quad \mathrm{S} 19$

Figure S14: ${ }^{13} \mathrm{C}\{1 \mathrm{H}\}$ NMR spectra of 2-OMe $\left(100 \mathrm{MHz}, \mathrm{CDCl}_{3}\right) \quad$ S20

Figure S15: ${ }^{\mathrm{H}} \mathrm{HMR}$ spectra of 2-NHMe $\left(400 \mathrm{MHz}, \mathrm{CDCl}_{3}\right) \quad \mathrm{S} 21$

Figure S16: ${ }^{13} \mathrm{C}\{1 \mathrm{H}\} \mathrm{NMR}$ spectra of 2-NHMe $\left(100 \mathrm{MHz}, \mathrm{CDCl}_{3}\right)$

Figure S17: ${ }^{1 \mathrm{H}} \mathrm{NMR}$ spectra of $9\left(600 \mathrm{MHz}, \mathrm{CDCl}_{3}\right) \quad$ S23

Figure S18: ${ }^{13} \mathrm{C}\{1 \mathrm{H}\} \mathrm{NMR}$ spectra of $9\left(100 \mathrm{MHz}, \mathrm{CDCl}_{3}\right) \quad \mathrm{S} 24$

Table S1: Spectral Properties of $5 \quad$ S25

Figure S19: Normalized Absorbances of 1-NHMe in various solvents $\quad$ S25

Figure S20: Normalized Emissions of 1-NHMe in various solvents $\quad$ S25

Figure S21: Normalized Absorbances of 1-OMe in various solvents $\quad$ S26

Figure S22: Normalized Emissions of 1-OMe in various solvents $\quad$ S26

Figure S23: Normalized Absorbances of 2-NHMe in various solvents $\quad$ S27

Figure S24: Normalized Emissions of 2-NHMe in various solvents $\quad$ S27

Computation Details $\quad$ S28

Figure S25: Calculated vertical excitation energies for $S_{1}$ and $S_{2} \quad S 28$

Figure S26: Calculated excitation energies for 1-NHMe and 2-NHMe along the amine S29

pyramidalization coordinate

Figure S27: Excited-state structures of 1-NHMe and 2-NHMe S29

$\begin{array}{ll}\text { Optimized Coordinates } & \text { S29 }\end{array}$

References $\quad$ S33 


\section{MATERIALS AND GENERAL METHODS}

Unless otherwise specified, all commercial products and reagents were used as purchased, without further purification. Analytical thin-layer chromatography (TLC) and flash chromatography of all reactions was performed on silica gel $(40 \mu \mathrm{m})$ purchased from Grace Davison. All solvents used for photophysical experiments were reagent grade. HPLC separation was performed using a Waters 2545 Binary Gradient Module with Water 2998 photodiode array detector using a $10.0 \mu \mathrm{m}$ C18 Sunfire column from Waters.

NMR Spectroscopy: ${ }^{1 \mathrm{H}}$ and ${ }^{13} \mathrm{C}$ NMR spectra for all compounds were acquired in deuterated solvents (as indicated) on a Bruker Spectrometer at the field strengths reported in the text. The chemical shift data are reported in units of $\delta(\mathrm{ppm})$ relative to residual solvent.

Absorption and Emission Spectroscopy: Fluorescence spectra were measured on an Agilent Technologies Car Eclipse Fluorescence Spectrophotometer using right-angle detection. Ultraviolet-visible absorption spectra were measured with an Agilent Technologies Cary 8454 UV-Vis Diode Array System and corrected for background signal with a cuvette containing the same solvent used for analysis.

Fluorescent quantum yields were determined relative to the fluorescence standard reported in the text and are corrected for solvent refractive index and absorption differences at the excitation wavelength. 
<smiles>Cc1cc(F)c(C(=O)O)c(N)c1[N+](=O)[O-]</smiles>

\section{Synthesis of 4}

Compound 4 has been previously reported, ${ }^{1}$ 2,5-difluoro-3-nitro benzoic acid (5 g, $25 \mathrm{mmol}, 1$ eq.) was dissolved into $250 \mathrm{~mL}$ of conc. $\mathrm{NH}_{3} \mathrm{OH}$ and stirred for $90 \mathrm{~min}$. The solvent was removed under reduced pressure to provide an orange-yellow solid that was re-dissolved in $\mathrm{H}_{2} \mathrm{O}$ at $90^{\circ} \mathrm{C}$. $1 \mathrm{M} \mathrm{HCl}$ was added dropwise until the solution was approximately $\mathrm{pH} 2$ to produce a yellow precipitate that was collected by vacuum filtration. The solid was dried overnight under vacuum and was used without further purification (4 g, 80\%). Compound 4 can be further purified with repeated silica gel chromatography (1:5 EtOAc: Hexanes). Spectra of purified spectra provided in Figure S1 and S2.

${ }^{1} \mathrm{H}$ NMR (DMSO- $\left.\mathrm{d}_{6}, 400 \mathrm{MHz}\right) \delta 8.28(\mathrm{~m}, 3 \mathrm{H}), 6.57(\mathrm{t}, J=9.9 \mathrm{~Hz}, 1 \mathrm{H})$.

${ }^{13} \mathrm{C}\{1 \mathrm{H}\}$ NMR (DMSO-d, $\left.100 \mathrm{MHz}\right) \delta 166.4\left(\mathrm{~d}, J_{\mathrm{C}-\mathrm{F}}=263.9 \mathrm{~Hz}\right), 166.2\left(\mathrm{~d}, J_{\mathrm{c}-\mathrm{F}}=2.5 \mathrm{~Hz}\right), 147.6\left(\mathrm{~d}, \mathrm{~J}_{\mathrm{C}-\mathrm{F}}=\right.$ 7.3 Hz), $132.6\left(\mathrm{~d}, J_{\mathrm{C}-\mathrm{F}}=14.5 \mathrm{~Hz}\right), 129.0\left(\mathrm{~d}, J_{\mathrm{C}-\mathrm{F}}=1.1 \mathrm{~Hz}\right), 104.8\left(\mathrm{~d}, J_{\mathrm{C}-\mathrm{F}}=14.9 \mathrm{~Hz}\right), 103.9\left(\mathrm{~d}, J_{\mathrm{C}-\mathrm{F}}=26.7\right.$ $\mathrm{Hz})$

LRMS (ESI) m/z: [M - H]- Calculated for $\mathrm{C}_{7} \mathrm{H}_{4} \mathrm{FN}_{2} \mathrm{O}_{4} 199$ amu; found $199 \mathrm{amu}$.<smiles>Nc1c(F)ccc([N+](=O)[O-])c1C(=O)O</smiles><smiles>c1ccoc1</smiles><smiles>O=[N+]([O-])c1ccc(F)c2c1C1C=CC2O1</smiles>

\section{Synthesis of 5}

Compound 4 (1.0 g, 5 mmol, 1 eq.) was dissolved into furan $(50 \mathrm{ml})$ under $\mathrm{N}_{2}$ in a 3-neck flask fitted with a condenser and other two ports were sealed with septa. TFA $(7 \mu \mathrm{L}, 0.05 \mathrm{mmol}, .01 \mathrm{eq}$.) and $t$-BuONO (2.5 $\mathrm{mL}, 20 \mathrm{mmol}, 4$ eq.) were added via syringe. The mixture was refluxed for $12 \mathrm{~h}$. After cooling the mixture was concentrated under reduced pressure. The residue was purified via silica gel chromatography (1:5 EtOAc: Hexanes) to yield 5 as a yellow solid $(0.38 \mathrm{~g}, 37 \%)$.

${ }^{1} \mathrm{H} \mathrm{NMR}\left(\mathrm{CDCl}_{3}, 400 \mathrm{MHz}\right) \delta 7.80(\mathrm{dd}, J=9.1,3.8 \mathrm{~Hz}, 1 \mathrm{H}), 7.21(\mathrm{dd}, J=5.5,1.8 \mathrm{~Hz}, 1 \mathrm{H}), 7.19(\mathrm{dd}, J=$ 5.5, $1.7 \mathrm{~Hz}, 1 \mathrm{H}), 6.88(\mathrm{dd}, J=7.1,2.0 \mathrm{~Hz}, 1 \mathrm{H}), 6.54(\mathrm{~m}, 1 \mathrm{H}), 6.07(\mathrm{~m}, 1 \mathrm{H})$.

${ }^{13} \mathrm{C}\{1 \mathrm{H}\} \mathrm{NMR}\left(\mathrm{CDCl}_{3}, 100 \mathrm{MHz}\right) \delta 158.2\left(\mathrm{~d}, J_{\mathrm{C}-\mathrm{F}}=255 \mathrm{~Hz}\right), 151.4\left(\mathrm{~d}, J_{\mathrm{C}-\mathrm{F}}=7 \mathrm{~Hz}\right), 143.9,142.3,139\left(\mathrm{~d}, J_{\mathrm{C}-\mathrm{F}}\right.$ $=3 \mathrm{~Hz}), 137.2\left(\mathrm{~d}, J_{\mathrm{C}-\mathrm{F}}=24 \mathrm{~Hz}\right), 123.0\left(\mathrm{~d}, \mathrm{~J}_{\mathrm{C}-\mathrm{F}}=8 \mathrm{~Hz}\right), 115.8\left(\mathrm{~d}, J_{\mathrm{C}-\mathrm{F}}=25 \mathrm{~Hz}\right), 83.3,79.4\left(\mathrm{~d}, \mathrm{~J}_{\mathrm{C}-\mathrm{F}}=1 \mathrm{~Hz}\right)$ HRMS (ESI-TOF) m/z: [M - H]- Calcd for C10H6NFO3, 206.0253 found 206.0260.<smiles>O=[N+]([O-])c1ccc(F)c2c1C1C=CC2O1</smiles>

\section{Synthesis of 8}

Compound 5 (103.5 mg, $0.5 \mathrm{mmol}, 1$ eq.) and tetrazine 6 (118 mg, $0.5 \mathrm{mmol}, 2$ eq.) were dissolved in DMF $(5 \mathrm{~mL})$. The mixture was stirred at room temp. for $48 \mathrm{~h}$ then concentrated under reduced pressure and purified with silica gel chromatography (2:8 EtOAc: Hexanes) to yield 8 as a yellow solid (0.046 g $51.5 \%)$. ${ }^{1} \mathrm{H} \mathrm{NMR}\left(\mathrm{CDCl}_{3}, 400 \mathrm{MHz}\right) \delta 8.69(\mathrm{t}, J=2.1 \mathrm{~Hz}, 1 \mathrm{H}), 8.34(\mathrm{~d}, J=1.6 \mathrm{~Hz}, 1 \mathrm{H}), 8.13(\mathrm{dd}, J=8.0,4.0 \mathrm{~Hz}$, $1 \mathrm{H}), 6.65(\mathrm{~m}, 1 \mathrm{H})$,

${ }^{13} \mathrm{C}\{1 \mathrm{H}\} \mathrm{NMR}\left(\mathrm{CDCl}_{3}, 100 \mathrm{MHz}\right) \delta 160.1\left(\mathrm{~d}, J_{\mathrm{C}-\mathrm{F}}=267 \mathrm{~Hz}\right), 138.8\left(\mathrm{~d}, \mathrm{~J}_{\mathrm{C}-\mathrm{F}}=2 \mathrm{~Hz}\right), 136.1\left(\mathrm{~d}, \mathrm{~J}_{\mathrm{C}-\mathrm{F}}=4 \mathrm{~Hz}\right)$, $127.1\left(\mathrm{~d}, \mathrm{~J}_{\mathrm{C}-\mathrm{F}}=10 \mathrm{~Hz}\right), 117.9,117.7,117.6,104.9\left(\mathrm{~d}, \mathrm{~J}_{\mathrm{C}-\mathrm{F}}=21 \mathrm{~Hz}\right)$.

HRMS (ESI-TOF) m/z: [M + H]+ Calcd for $\mathrm{C}_{8} \mathrm{H}_{5} \mathrm{FNO}_{3}$, Calcd 182.0248, found 182.0241 . 
<smiles>COc1cc2c(c([N+](=O)[O-])c1)C1C=CC2O1</smiles>

\section{Synthesis of 10-OMe}

Based on a previously reported procedure, ${ }^{2}$ compound 5 (54 mg, $0.3 \mathrm{mmol}, 1 \mathrm{eq}$ ) was dissolved in $\mathrm{MeOH}$ $(0.5 \mathrm{~mL}, 0.6 \mathrm{M})$ and cooled to $0^{\circ} \mathrm{C} . \mathrm{KOH}(25 \mathrm{mg}, 0.45 \mathrm{mmol}, 1.5 \mathrm{eq})$ was added and the mixture was stirred at $60^{\circ} \mathrm{C}$. After TLC indicated full conversion $(2 \mathrm{~h})$, the solution was diluted with $\mathrm{H}_{2} \mathrm{O}(10 \mathrm{~mL})$ and the aqueous layer was extracted with EtOAc $(3 \times 10 \mathrm{~mL})$. The combined organic layers were dried over $\mathrm{Na}_{2} \mathrm{SO}_{4}$ and concentrated in vacuo. The crude product was purified via silica gel chromatography (1:4 EtOAc: Hexanes) to yield 10-OMe as a yellow solid (40 $\mathrm{mg}, 69 \%$ yield).

${ }_{1}^{1} \mathrm{H} \mathrm{NMR}\left(\mathrm{CDCl}_{3}, 400 \mathrm{MHz}\right) \delta 7.80(\mathrm{~d}, J=9.08 \mathrm{~Hz}, 1 \mathrm{H}), 7.17(\mathrm{t}, J=0.96 \mathrm{~Hz}, 2 \mathrm{H}), 6.71$ (d, J=9.08 Hz, $1 \mathrm{H}), 6.51$ (q, $J=0.96 \mathrm{~Hz}, 1 \mathrm{H}), 6.00(\mathrm{q}, J=0.96 \mathrm{~Hz}, 1 \mathrm{H}), 3.94(\mathrm{~s}, 3 \mathrm{H})$.

${ }_{12} \mathrm{C}\{1 \mathrm{H}\} \mathrm{NMR}\left(\mathrm{CDCl}_{3}, 100 \mathrm{MHz}\right) \delta 156.69,150.18,144.24,142.25,138.48,136.84,122.82,110.79$, 83.26, 79.85, 56.15.

HRMS (ESI-TOF) m/z: [M - H]- Calcd for $\mathrm{C}_{11} \mathrm{H}_{9} \mathrm{NO}_{4}, 218.0458$ found 218.0459.<smiles>CNc1cc(C2C3C=CC(O3)C2C)c2c(c1[N+](=O)[O-])C1C=CC2O1</smiles>

\section{Synthesis of 10-NHMe}

Compound 5 (0.145 g, $0.7 \mathrm{mmol}, 1 \mathrm{eq})$ and $\mathrm{K}_{2} \mathrm{CO}_{3}(0.386 \mathrm{~g}, 2.8 \mathrm{mmol}, 4 \mathrm{eq})$ were suspended in DMSO $(1.4 \mathrm{~mL}, 0.5 \mathrm{M})$ and $2 \mathrm{M} \mathrm{MeNH}_{2}$ in THF $(0.35 \mathrm{~mL}, 0.7 \mathrm{mmol}, 1 \mathrm{eq})$ was added. The mixture was stirred at $60^{\circ} \mathrm{C}$ for $2 \mathrm{~h}$. The solution was diluted with $\mathrm{H}_{2} \mathrm{O}(20 \mathrm{~mL})$ and $1 \mathrm{M} \mathrm{HCl}(1 \mathrm{~mL})$ and the aqueous phase was extracted with EtOAc $(3 \times 20 \mathrm{~mL})$. The combined organic phases were dried over $\mathrm{Na}_{2} \mathrm{SO}_{4}$, concentrated under reduced pressure, and the residue was purified via silica gel chromatography (1:1 EtOAc: Hexanes) to yield 10-NHMe as an orange solid $(0.0916 \mathrm{~g}, 60 \%$ yield). ${ }_{1} \mathrm{H} \mathrm{NMR}\left(\mathrm{CDCl}_{3}, 400 \mathrm{MHz}\right) \delta 7.76(\mathrm{~d}, J=9.08 \mathrm{~Hz}, 1 \mathrm{H}), 7.20(\mathrm{dd}, J=5.48,1.96 \mathrm{~Hz}, 1 \mathrm{H}), 7.14(\mathrm{dd}, J=$ $5.48,1.96 \mathrm{~Hz}, 1 \mathrm{H}) 6.57(\mathrm{~s}, 1 \mathrm{H}), 6.39(\mathrm{~d}, J=9.08 \mathrm{~Hz}, 1 \mathrm{H}), 5.87(\mathrm{~s}, 1 \mathrm{H}), 4.33(\mathrm{~s}, 1 \mathrm{H}), 3.01(\mathrm{~s}, 3 \mathrm{H})$. ${ }^{13} \mathrm{C}\{1 \mathrm{H}\} \mathrm{NMR}\left(\mathrm{CDCl}_{3}, 100 \mathrm{MHz}\right) \delta 149.62,146.57,143.60,142.56,134.24,133.68,123.19,109.85$, 83.49, 79.59, 30.59.

HRMS (ESI-TOF) m/z: [M - H] - Calcd for $\mathrm{C}_{11} \mathrm{H}_{10} \mathrm{~N}_{2} \mathrm{O}_{3}, 217.0618$ found 217.0619.<smiles>O=[N+]([O-])c1ccc(F)c2c1C1C=CC2O1</smiles>

\section{Synthesis of $10-\mathrm{R}^{3}$}

Compound $5(0.108 \mathrm{~g}, 0.7 \mathrm{mmol}, 1 \mathrm{eq})$ and $\mathrm{Et}_{3} \mathrm{~N}(0.33 \mathrm{~mL}, 2.4 \mathrm{mmol}, 4 \mathrm{eq})$ were dissolved in $\mathrm{MeCN}(3$ $\mathrm{mL})$. The mixture was stirred at $60^{\circ} \mathrm{C}$ for $16 \mathrm{hrs}$. The reaction was diluted with $\mathrm{H}_{2} \mathrm{O}(15 \mathrm{~mL})$ and extracted with EtOAc $(3 \times 20 \mathrm{~mL})$. The combined organic layers were dried over $\mathrm{Na}_{2} \mathrm{SO}_{4}$, concentrated under reduced pressure, and the residue was purified via silica gel chromatography (1:4 EtOAc:Hexanes) to yield $\mathbf{1 0}-\mathbf{R}^{3}$ as an orange solid. (0.095 $\mathrm{g}, 73 \%$ yield) 
${ }^{1} \mathrm{H}$ NMR $\left(400 \mathrm{MHz}, \mathrm{CDCl}_{3}\right) \delta 7.67(\mathrm{~d}, J=9.0 \mathrm{~Hz}, 1 \mathrm{H}), 7.12(\mathrm{dt}, J=5.5,1.5 \mathrm{~Hz}, 1 \mathrm{H}), 7.08(\mathrm{dt}, J=5.4,1.5 \mathrm{~Hz}$, $1 \mathrm{H}), 6.51(\mathrm{~m}, 1 \mathrm{H}), 6.06(\mathrm{dt}, J=9.0 \mathrm{~Hz}, 1 \mathrm{H}), 5.90(\mathrm{~m}, 1 \mathrm{H}), 4.19(\mathrm{q}, J=7.4 \mathrm{~Hz}, 2 \mathrm{H}), 4.12(\mathrm{q}, J=7.4 \mathrm{~Hz}, 2 \mathrm{H})$, $2.49(\mathrm{~m}, 2 \mathrm{H})$.

${ }^{13} \mathrm{C}\{1 \mathrm{H}\} \mathrm{NMR}\left(100 \mathrm{MHz}, \mathrm{CDCl}_{3}\right) \delta 150.3,147.8,144.6,141.8,134.1,132.1,122.6,110.6,82.8,80.8,53.0,17.0$

HRMS (ESI-TOF) m/z: [M+ H]+ Calcd for $\mathrm{C}_{13} \mathrm{H}_{12} \mathrm{~N}_{2} \mathrm{O}_{3}, 245.0921$ found 245.0916 .<smiles>COc1ccc([N+](=O)[O-])c2c1COC2C</smiles>

Synthesis of 2-OMe

Adapted from a previously reported procedure, ${ }^{3}$ compound $10-0 \mathrm{Me}(25 \mathrm{mg}, 0.11 \mathrm{mmol}, 1 \mathrm{eq}$.$) and the$ tetrazine (26 mg, $0.11 \mathrm{mmol}, 1$ eq.) were dissolved in $1.1 \mathrm{~mL}$ DMF and stirred at RT for $30 \mathrm{~min}$. The reaction was then concentrated under reduced pressure and the residue was purified by HPLC (gradient of 55:45 $\mathrm{H}_{2} \mathrm{O}: \mathrm{MeCN}$ to $35: 65 \mathrm{H}_{2} \mathrm{O}: \mathrm{MeCN}$ over $26 \mathrm{~min}$ ) to yield 2-OMe as a yellow solid. (11.4 $\mathrm{mg}, 53 \%$ yield).

${ }_{1}^{1} \mathrm{H} \mathrm{NMR}\left(\mathrm{CDCl}_{3}, 600 \mathrm{MHz}\right) \delta 8.56(\mathrm{~d}, J=1.8 \mathrm{~Hz}, 1 \mathrm{H}), 8.24(\mathrm{~d}, J=1.8 \mathrm{~Hz}, 1 \mathrm{H}), 8.16(\mathrm{~d}, J=8.1,1 \mathrm{H}) 6.18$ (d, $J=8.1 \mathrm{~Hz}, 1 \mathrm{H}), 4.05(\mathrm{~s}, 3 \mathrm{H})$.

${ }_{13} \mathrm{C}\{1 \mathrm{H}\} \operatorname{NMR}\left(100 \mathrm{MHz}, \mathrm{CDCl}_{3}\right) \delta 159.9,137.8,137.2,129.0,125.2,119.8,116.9,97.7,56.3$.

HRMS (ESI-TOF) m/z: [M + H]+ Calcd for $\mathrm{C}_{9} \mathrm{H}_{7} \mathrm{NO}_{4}, 194.0448$ found 194.0449.<smiles>CNc1ccc([N+](=O)[O-])c2c1C1C=CC2O1</smiles>

Synthesis of 2-NHMe

Adapted from a previously reported procedure, ${ }^{3}$ compound 10-NHMe ( $25 \mathrm{mg}, 0.12 \mathrm{mmol}, 1$ eq.) and tetrazine 6 (28 mg, $0.12 \mathrm{mmol}, 1$ eq.) were dissolved in $1.2 \mathrm{~mL}$ DMF and stirred at RT for $30 \mathrm{~min}$. The reaction was then concentrated under reduced pressure and the residue was purified by HPLC (gradient of 55:45 $\mathrm{H}_{2} \mathrm{O}$ : MeCN to 35:65 $\mathrm{H}_{2} \mathrm{O}$ : MeCN over $26 \mathrm{~min}$ ) to yield 2-NHMe as a dark red solid (11 mg, 47\% yield).

${ }^{1} \mathrm{H}$ NMR (DMSO-d $\left.6,600 \mathrm{MHz}\right) \delta 8.69(\mathrm{~s}, 1 \mathrm{H}), 8.55$ (broad s, $\left.1 \mathrm{H}\right), 8.53(\mathrm{~d}, J=1.8 \mathrm{~Hz}, 1 \mathrm{H}) 8.17$ (d, J= 8.6 $\mathrm{Hz}, 1 \mathrm{H}), 5.93(\mathrm{~d}, J=8.6 \mathrm{~Hz}, 1 \mathrm{H}) 3.00(\mathrm{~d}, J=4.1 \mathrm{~Hz}, 3 \mathrm{H})$.

${ }^{13} \mathrm{C}\{1 \mathrm{H}\} \mathrm{NMR}\left(100 \mathrm{MHz}, \mathrm{DMSO}-\mathrm{d}_{6}\right) \delta 151.0,139.7,137.4,133.5,125.3,118.0,117.1,96.2,30.0$.

HRMS (ESI-TOF) m/z: [M + H]+ Calcd for $\mathrm{C}_{9} \mathrm{H}_{8} \mathrm{~N}_{2} \mathrm{O}_{3}, 193.0608$ found 193.0601 . 


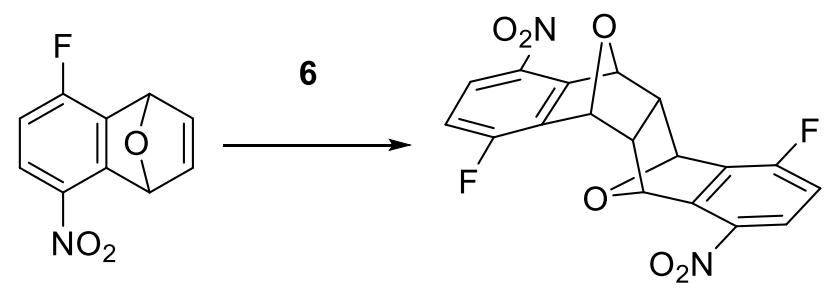

Synthesis of 9

Adapted from a previously reported procdure ${ }^{3}, 5(35 \mathrm{mg}, 0.16 \mathrm{mmol})$ was dissolved in chloroform (1.6 $\mathrm{mL}$ ) then 6 (37 mg, $0.16 \mathrm{mmol}$ ) was added. The suspension stirred at room temperature for 3 hours until starting material was consumed. The mixture was concentrated under reduced pressure and the residue was purified via silica gel chromatography (EtOAc: Hexane, 8:2) to give a white powder. (35 mg, 56\% yield).

${ }_{1}^{1} \mathrm{H} \mathrm{NMR}\left(\mathrm{CDCl}_{3}, 400 \mathrm{MHz}\right) \delta 8.07$ (dd, J=9.08, 3.88 Hz, 1H), 7.98 (dd, J=9.08, 3.88 Hz, 1H), 7.16 (dd, $\mathrm{J}=9.04,7.24 \mathrm{~Hz}, 1 \mathrm{H}), 7.05(\mathrm{dd}, \mathrm{J}=9.04,7.16 \mathrm{~Hz}, 1 \mathrm{H}), 6.16(\mathrm{dd}, \mathrm{J}=5.04,0.96 \mathrm{~Hz}, 1 \mathrm{H}), 5.80(\mathrm{~d}, \mathrm{~J}=1.92$ $\mathrm{Hz}, 1 \mathrm{H}), 5.56(\mathrm{~d}, \mathrm{~J}=1.92 \mathrm{~Hz}, 1 \mathrm{H}), 5.19(\mathrm{~s}, 1 \mathrm{H}), 3.14(\mathrm{~m}, 2 \mathrm{H})$

${ }_{13} \mathrm{C}\{1 \mathrm{H}\}$ NMR $\left(100 \mathrm{MHz}, \mathrm{CDCl}_{3}\right) \delta 159.6\left(\mathrm{~d}, \mathrm{~J}_{\mathrm{CF}}=256 \mathrm{~Hz}\right), 157.0\left(\mathrm{~d}, \mathrm{~J}_{\mathrm{CF}}=258 \mathrm{~Hz}\right), 146.4\left(\mathrm{~d}, \mathrm{~J}_{\mathrm{CF}}=6 \mathrm{~Hz}\right)$, 144.3(d, $\left.\mathrm{J}_{\mathrm{CF}}=2 \mathrm{~Hz}\right), 139.0,138.7,134.5\left(\mathrm{~d}, \mathrm{~J}_{\mathrm{CF}}=24 \mathrm{~Hz}\right), 131.8\left(\mathrm{~d}, \mathrm{~J}_{\mathrm{CF}}=22 \mathrm{~Hz}\right), 125.4$ (d, J J $125.0\left(\mathrm{~d}, \mathrm{~J}_{\mathrm{CF}}=9 \mathrm{~Hz}\right), 116.9\left(\mathrm{~d}, \mathrm{~J}_{\mathrm{CF}}=5 \mathrm{~Hz}\right), 116.7\left(\mathrm{~d}, \mathrm{~J}_{\mathrm{CF}}=5 \mathrm{~Hz}\right), 80.7,78.6,77.0\left(\mathrm{~d}, \mathrm{~J}_{\mathrm{CF}}=2 \mathrm{~Hz}\right), 74.8(\mathrm{~d}$, $\left.J_{\mathrm{CF}}=1 \mathrm{~Hz}\right), 49.9,48.3$.

HRMS (ESI-TOF) m/z: molecular ion not found. Please see $x$-ray crystal structure for structural confirmation. 
NMR SPECTRA
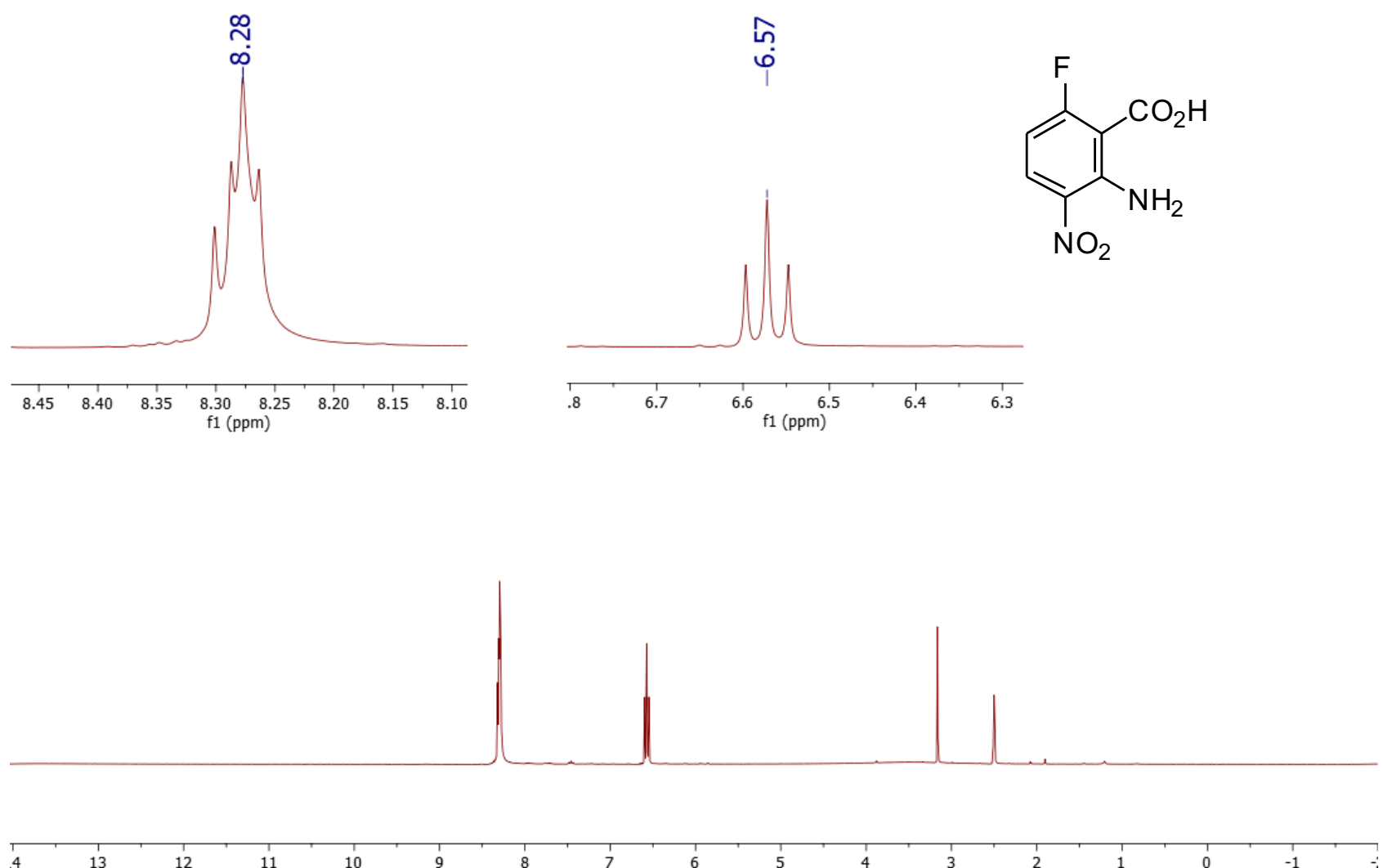

Figure $\mathrm{S} 1{ }^{1} \mathrm{H}$ NMR Spectra 4 in DMSO- $\mathrm{d}_{6}(400 \mathrm{MHz})$ 


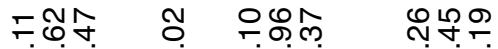

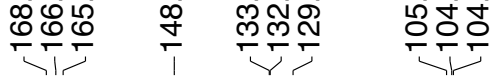<smiles>Nc1c(F)ccc([N+](=O)[O-])c1C(=O)O</smiles>

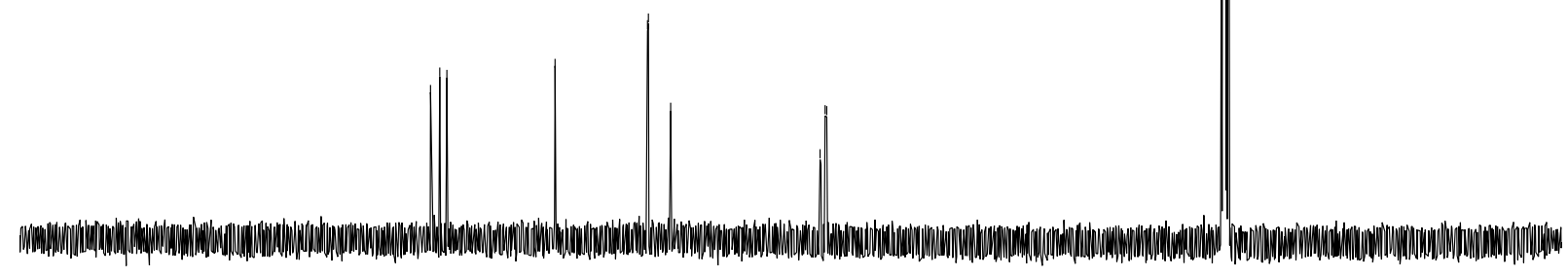

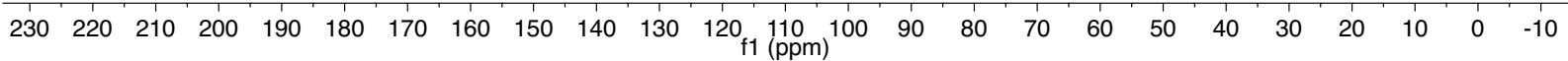

Figure S2 ${ }^{13} \mathrm{C}\{1 \mathrm{H}\}$ NMR Spectra 4 in DMSO-d $(100 \mathrm{MHz})$ 

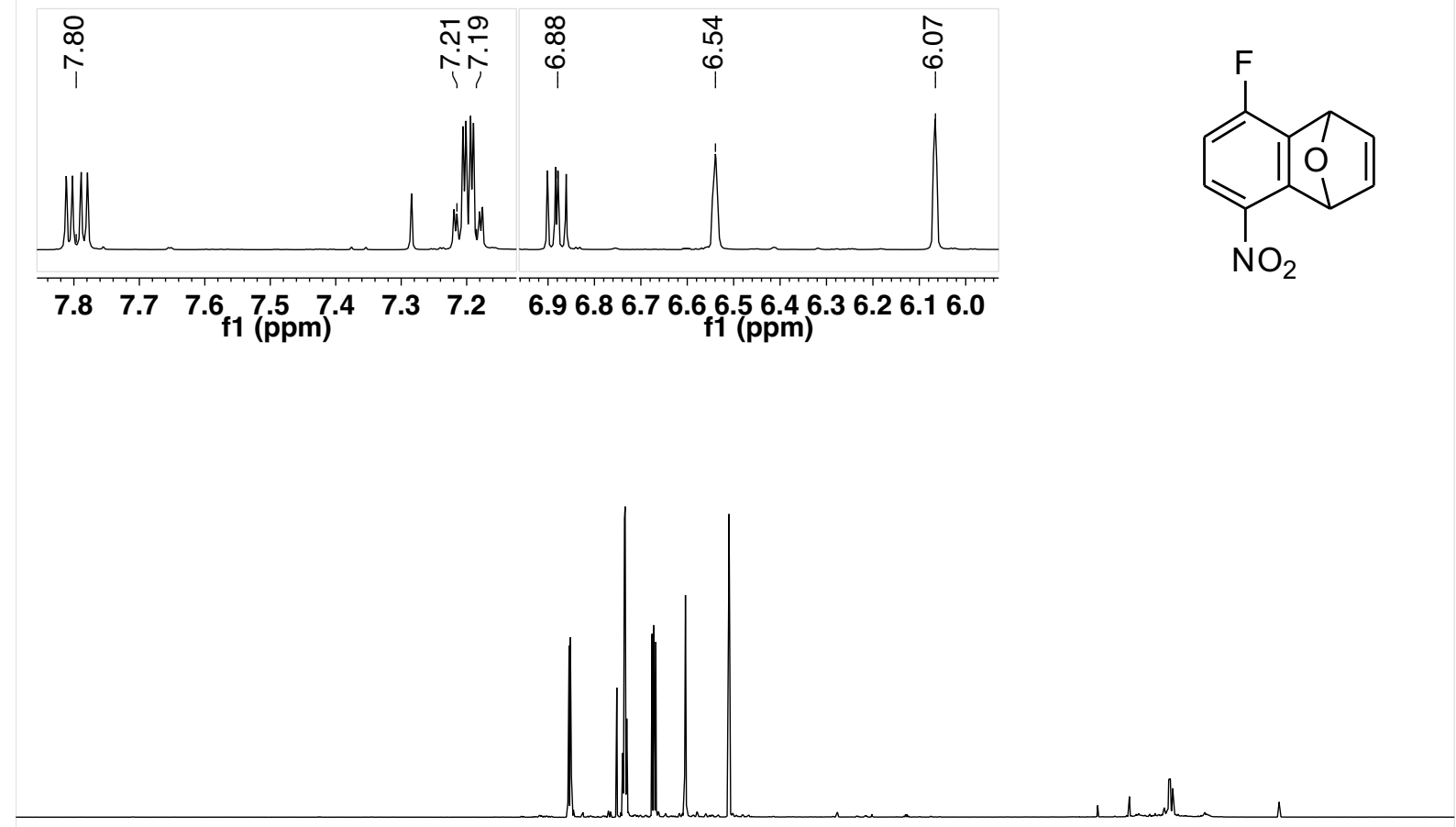

$13 \quad 12 \quad 11 \quad 10 \quad 9$

7 f1 $\underset{\text { (ppm) }}{6}$

Figure S3 ${ }^{1} \mathrm{H}$ NMR Spectra 5 in $\mathrm{CDCl}_{3}(400 \mathrm{MHz})$ 


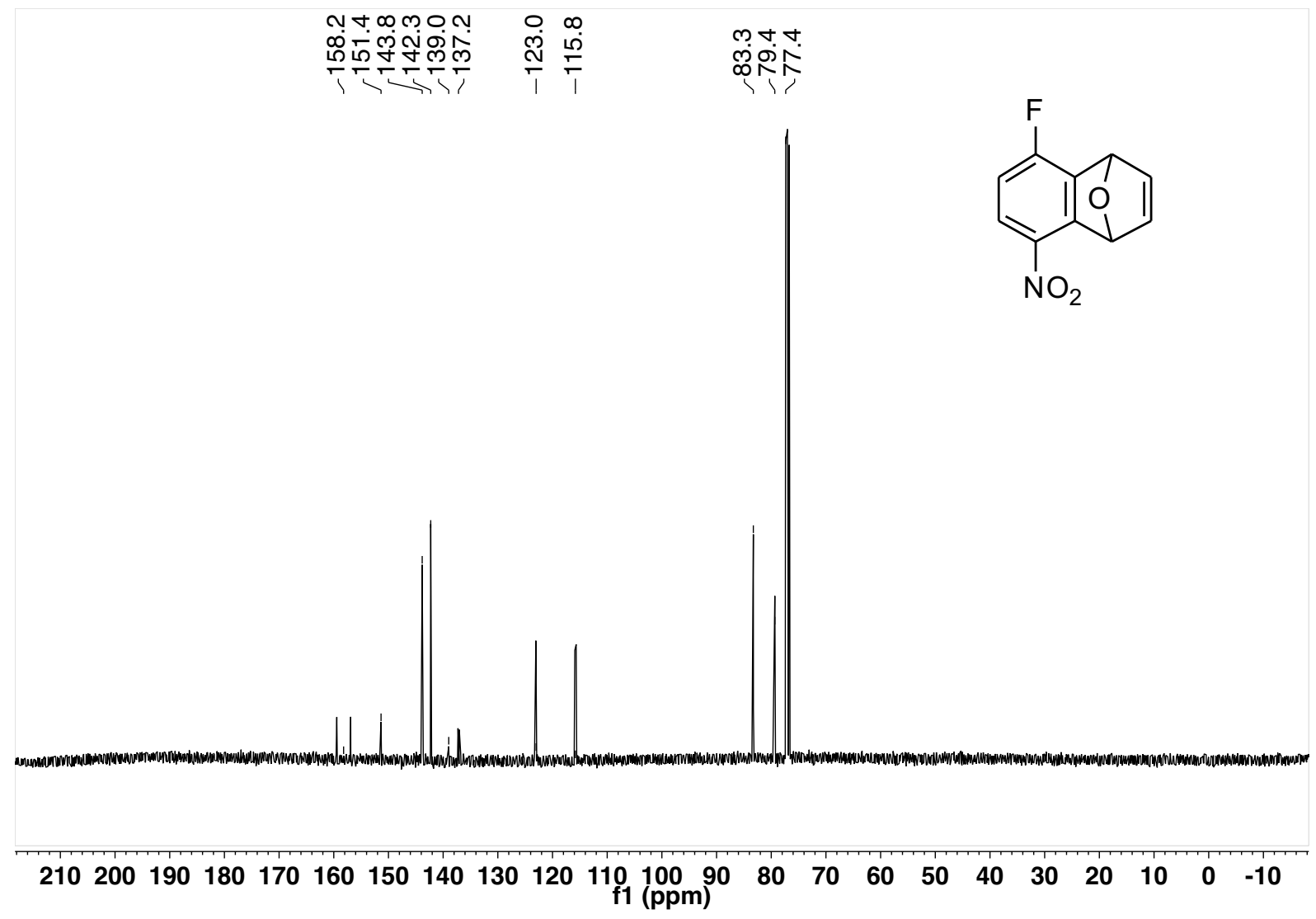

Figure $\mathrm{S} 4{ }^{13} \mathrm{C}\{1 \mathrm{H}\} \mathrm{NMR}$ Spectra 5 in $\mathrm{CDCl}_{3}(100 \mathrm{MHz})$ 

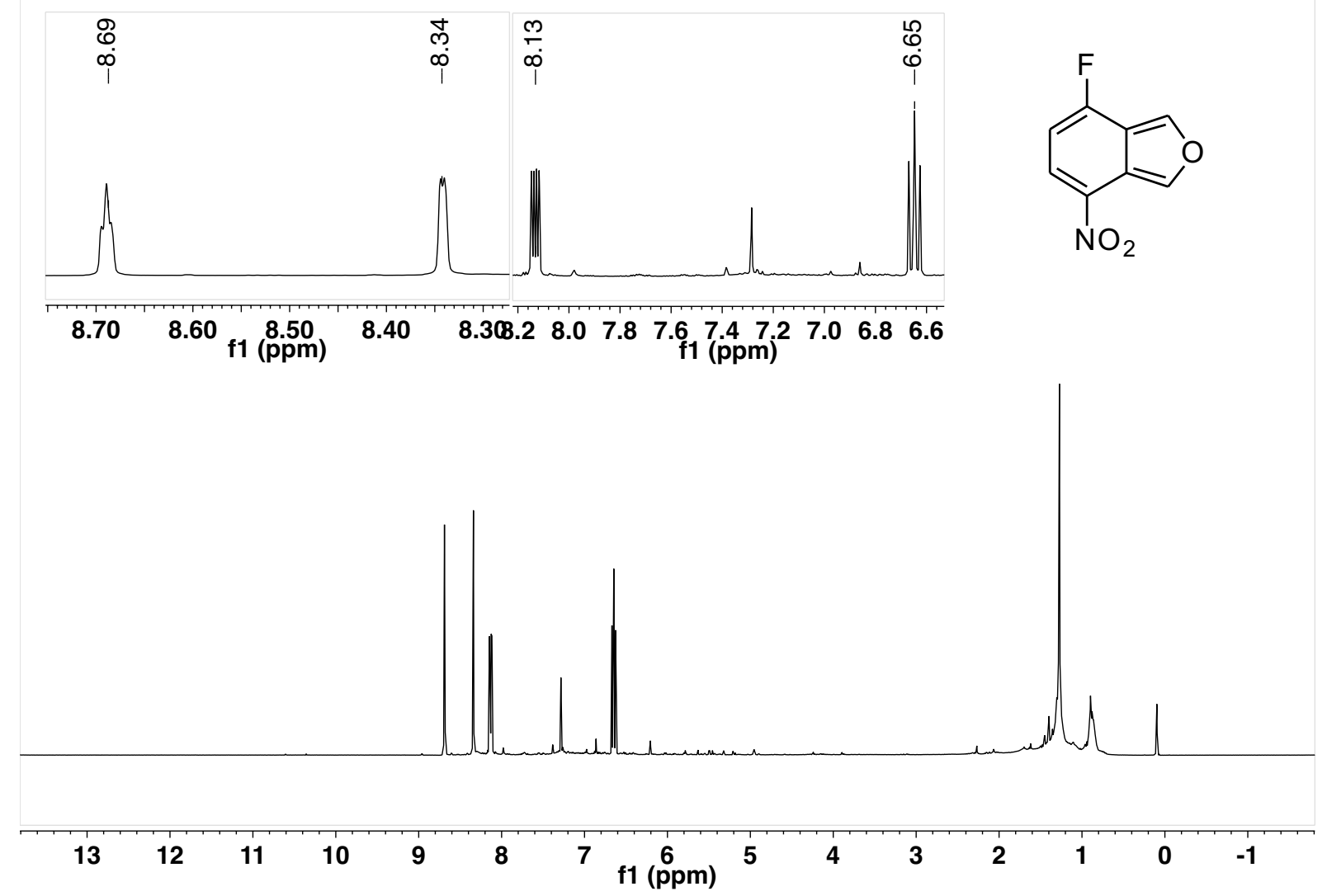

Figure S5 ${ }^{1} \mathrm{H}$ NMR Spectra 8 in $\mathrm{CDCl}_{3}(400 \mathrm{MHz})$ 


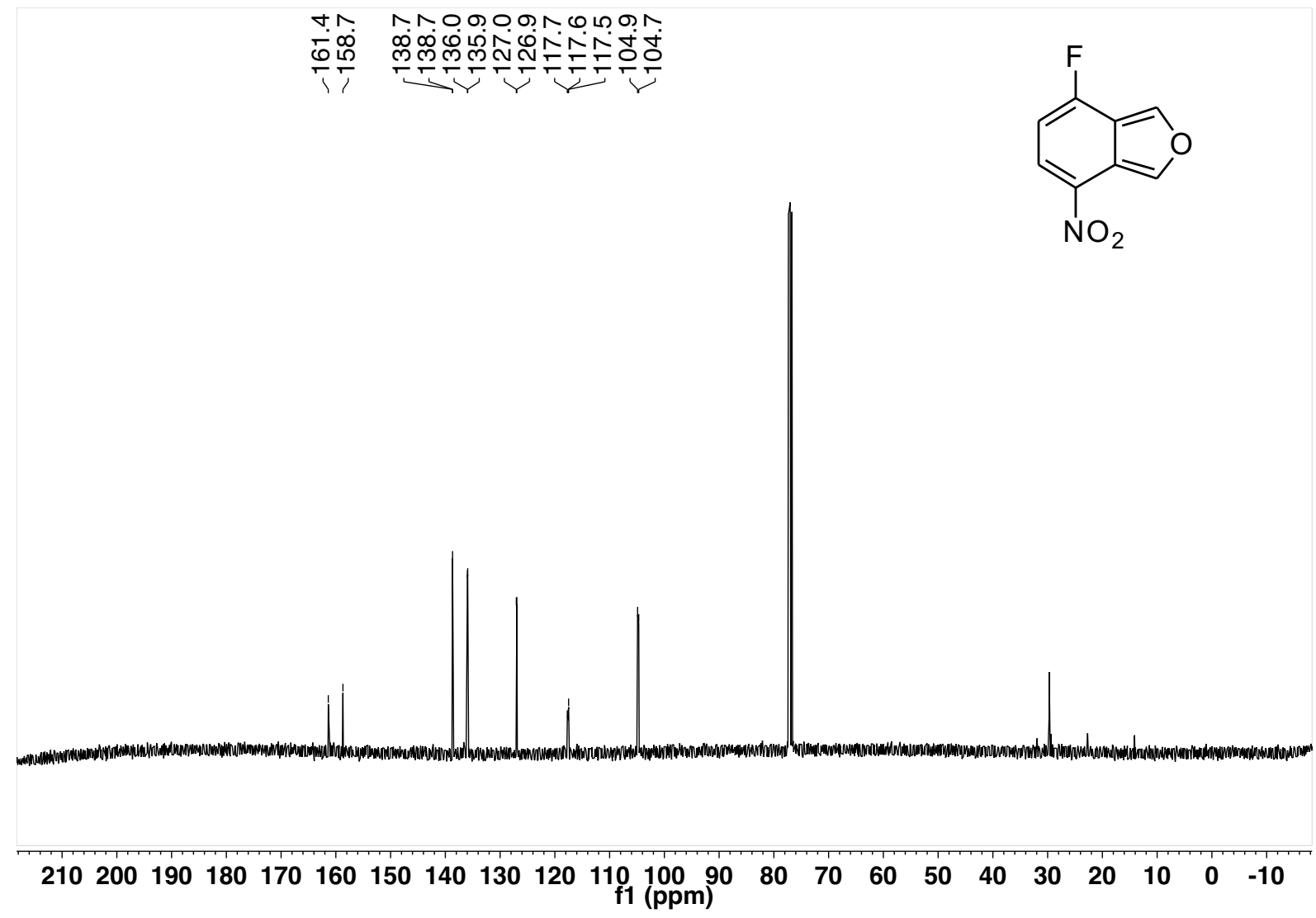

Figure $\mathrm{S} 6{ }^{13} \mathrm{C}\{1 \mathrm{H}\} \mathrm{NMR}$ Spectra 8 in $\mathrm{CDCl}_{3}(100 \mathrm{MHz})$ 


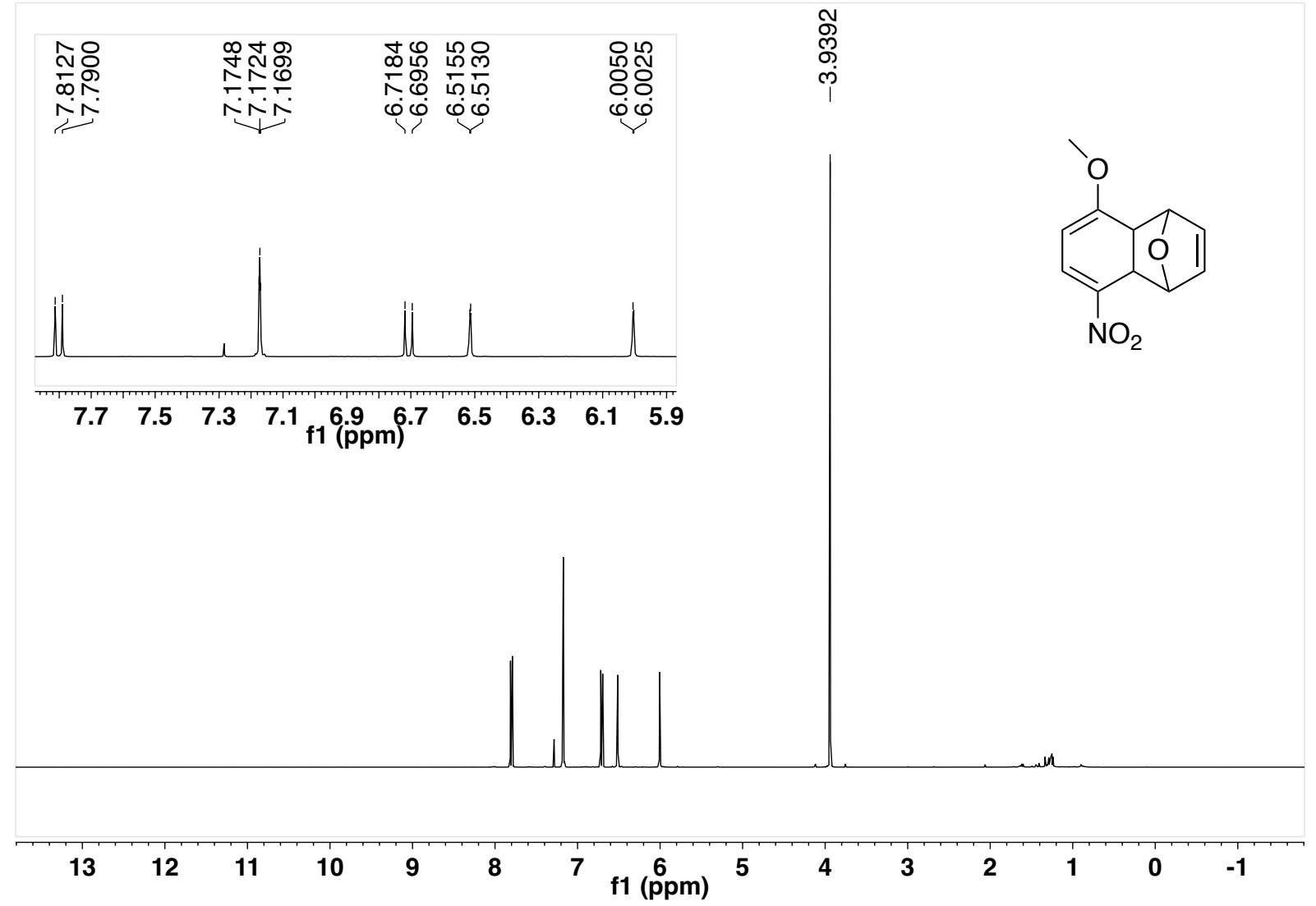

Figure $\mathrm{S} 7{ }^{1} \mathrm{H}$ NMR Compound 10-OMe in $\mathrm{CDCl}_{3}(400 \mathrm{MHz})$ 

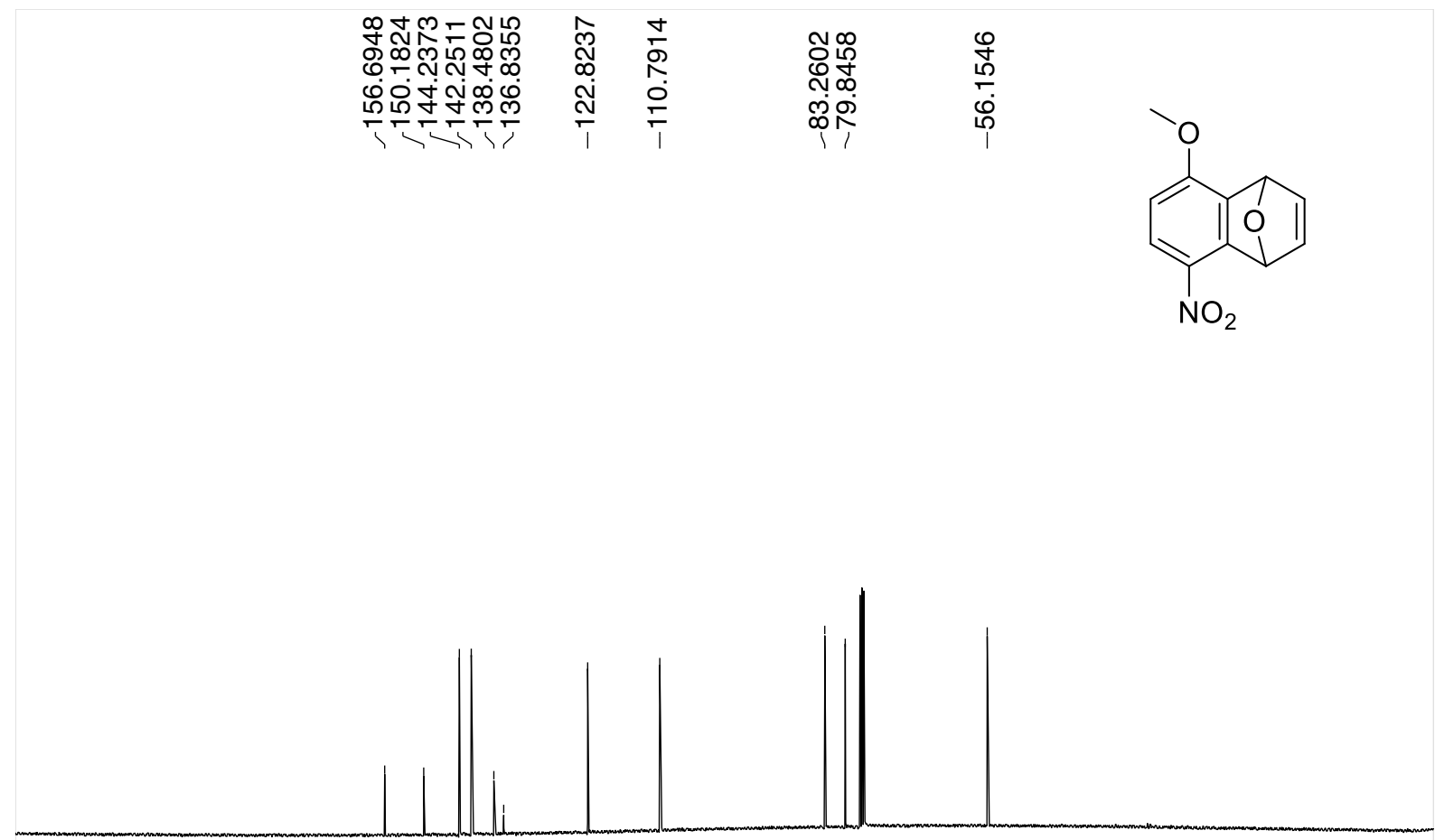

$\begin{array}{lllllllllllllllllll}210 & 200 & 190 & 180 & 170 & 160 & 150 & 140 & 130 & 120 & 110 \\ \mathrm{f} 1 & (\mathrm{ppm}) \\ (\mathrm{pp}) & 80 & 80 & 70 & 60 & 50 & 40 & 30 & 20 & 10 & 0 & -10\end{array}$

Figure $\mathrm{S} 8{ }^{13} \mathrm{C}\{1 \mathrm{H}\}$ NMR Compound 10-OMe in $\mathrm{CDCl}_{3}(100 \mathrm{MHz})$ 


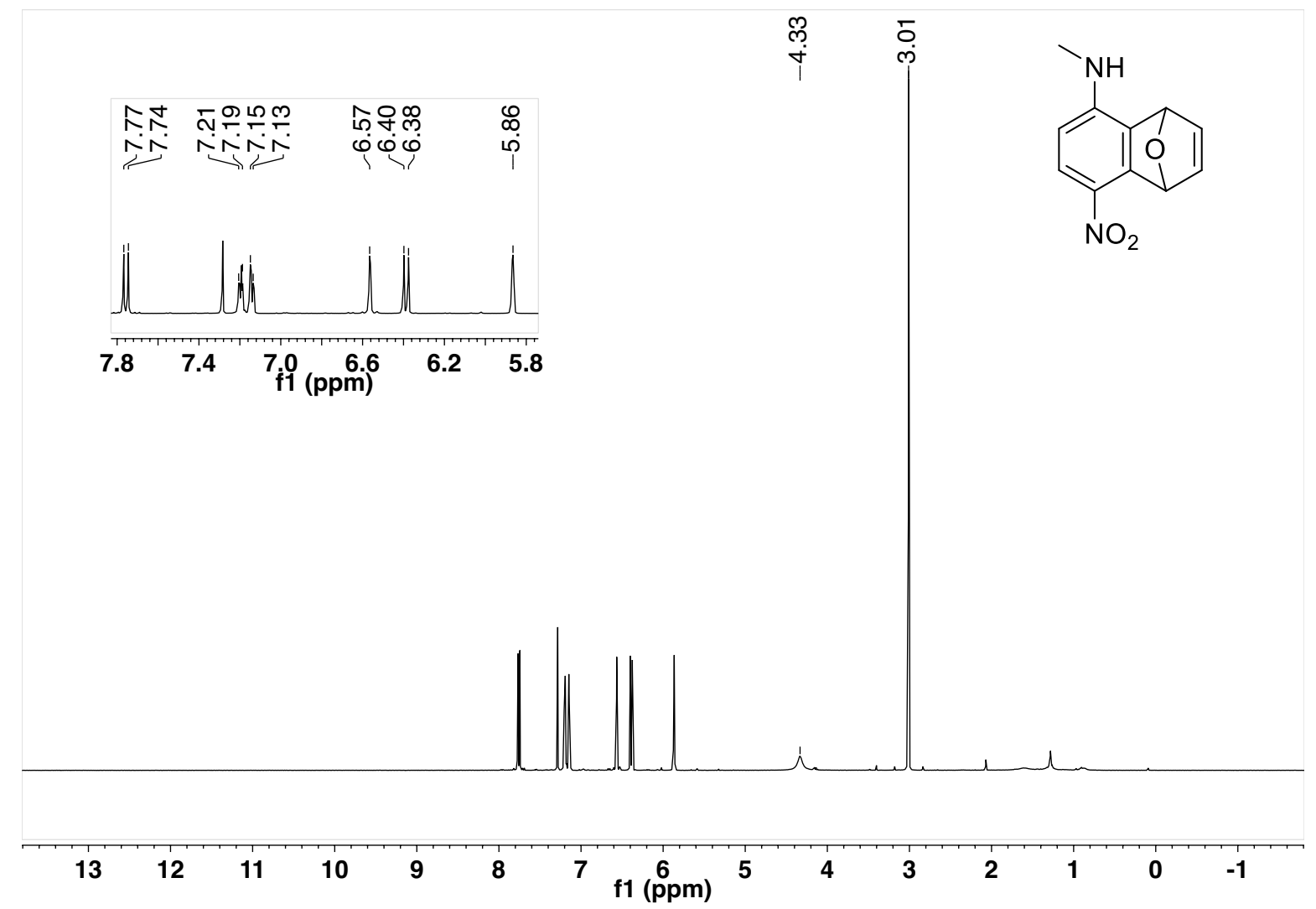

Figure S9 ${ }^{1} \mathrm{H}$ NMR Compound 10-NHMe in $\mathrm{CDCl}_{3}(400 \mathrm{MHz})$ 


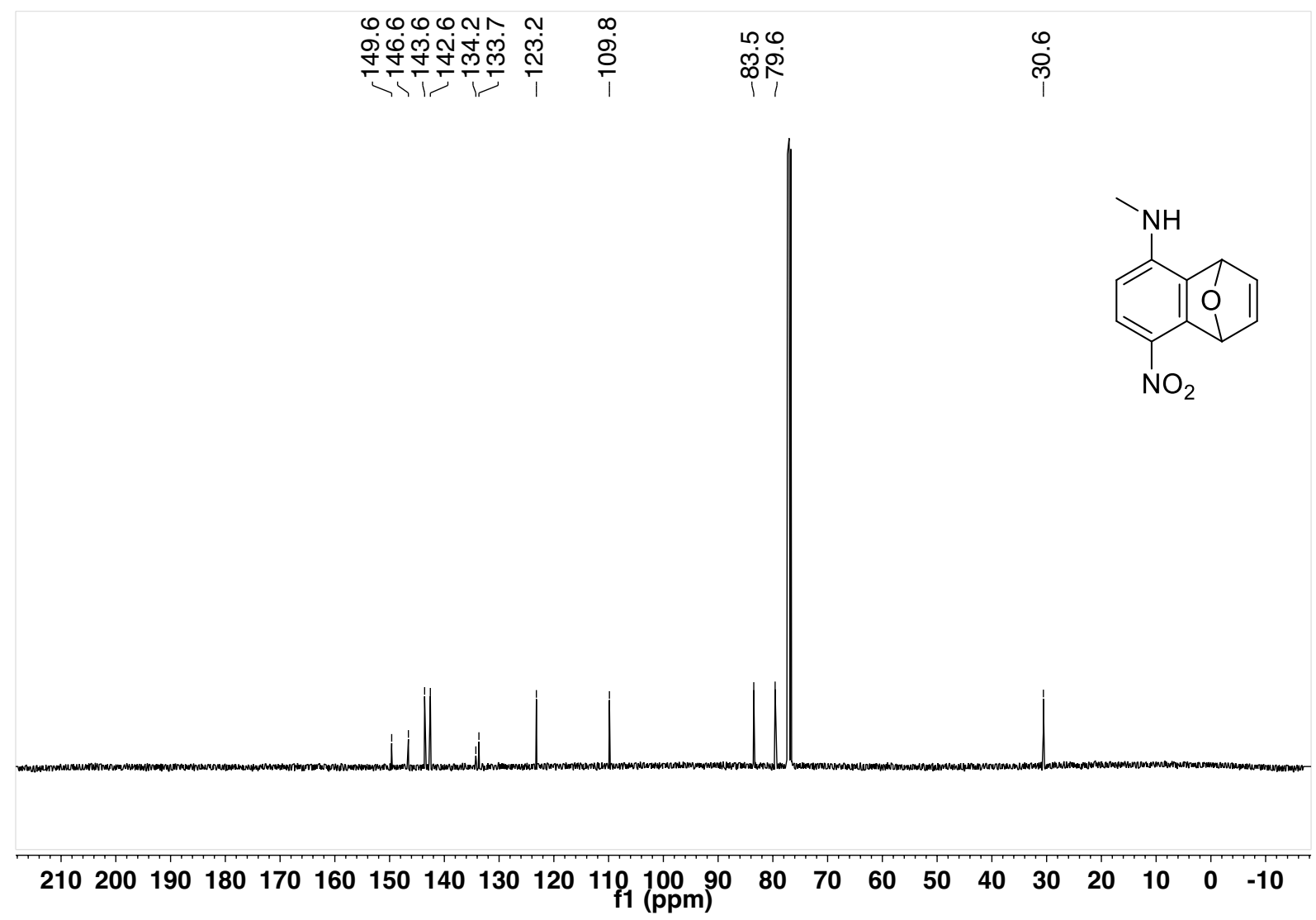

Figure $\mathrm{S} 10{ }^{13} \mathrm{C}\{1 \mathrm{H}\}$ NMR Compound $10-\mathrm{NHMe}$ in $\mathrm{CDCl}_{3}(100 \mathrm{MHz})$ 

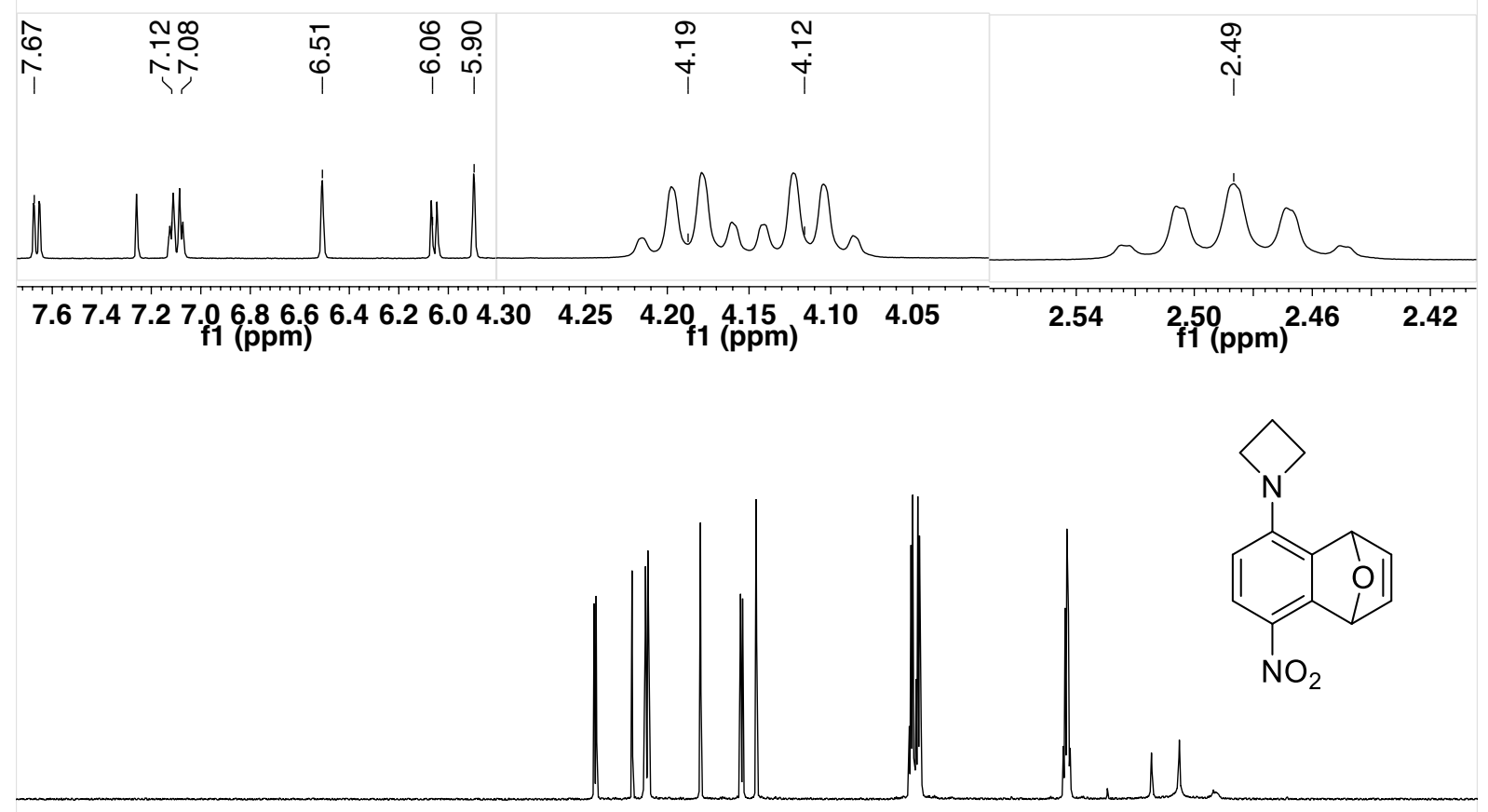

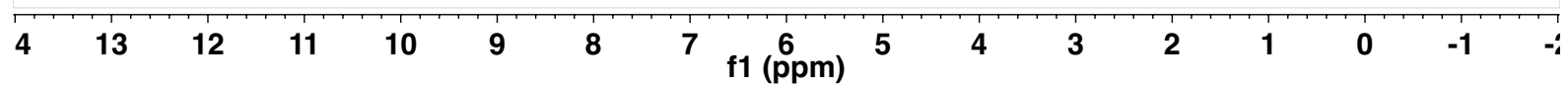

Figure $\mathrm{S} 11{ }^{1} \mathrm{H}$ NMR Compound $10-\mathbf{R}^{3}$ in $\mathrm{CDCl}_{3}(400 \mathrm{MHz})$ 


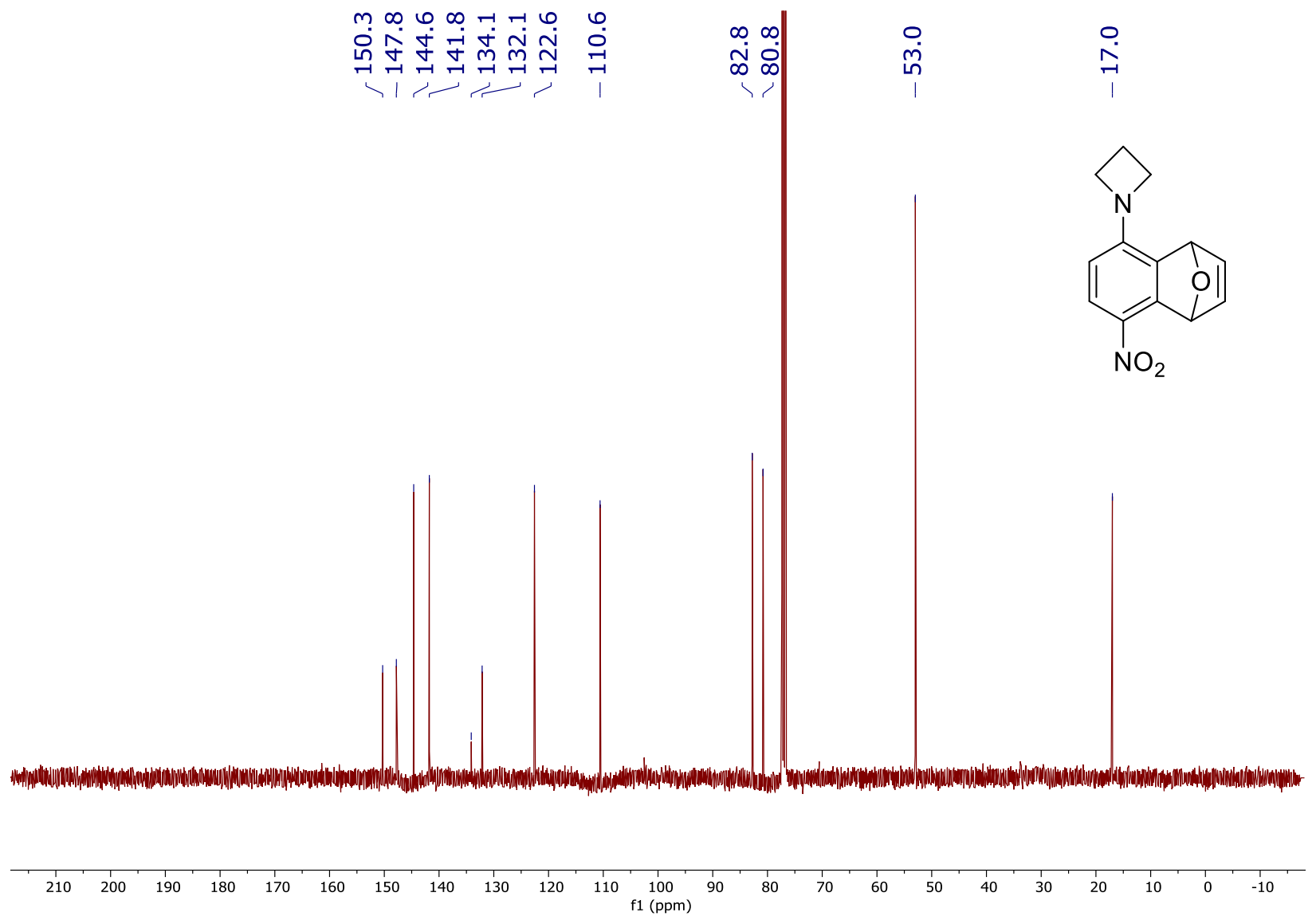

Figure $\mathrm{S} 12{ }^{13} \mathrm{C}\{1 \mathrm{H}\}$ NMR Compound $10-\mathrm{R}^{3}$ in $\mathrm{CDCl}_{3}(100 \mathrm{MHz})$ 


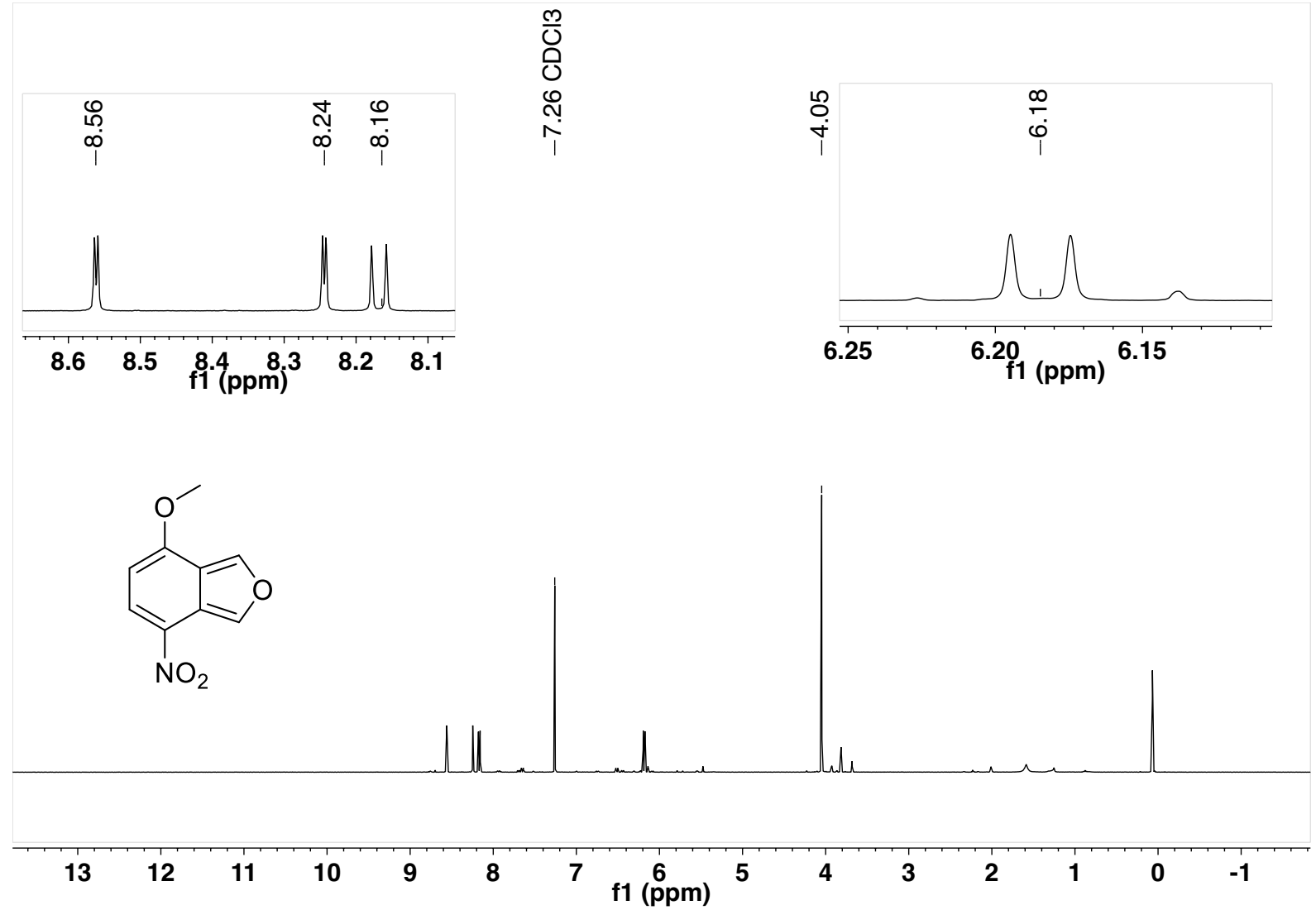

Figure S13 ${ }^{1} \mathrm{H}$ NMR Compound 2-OMe in $\mathrm{CDCl}_{3}(600 \mathrm{MHz})$ 


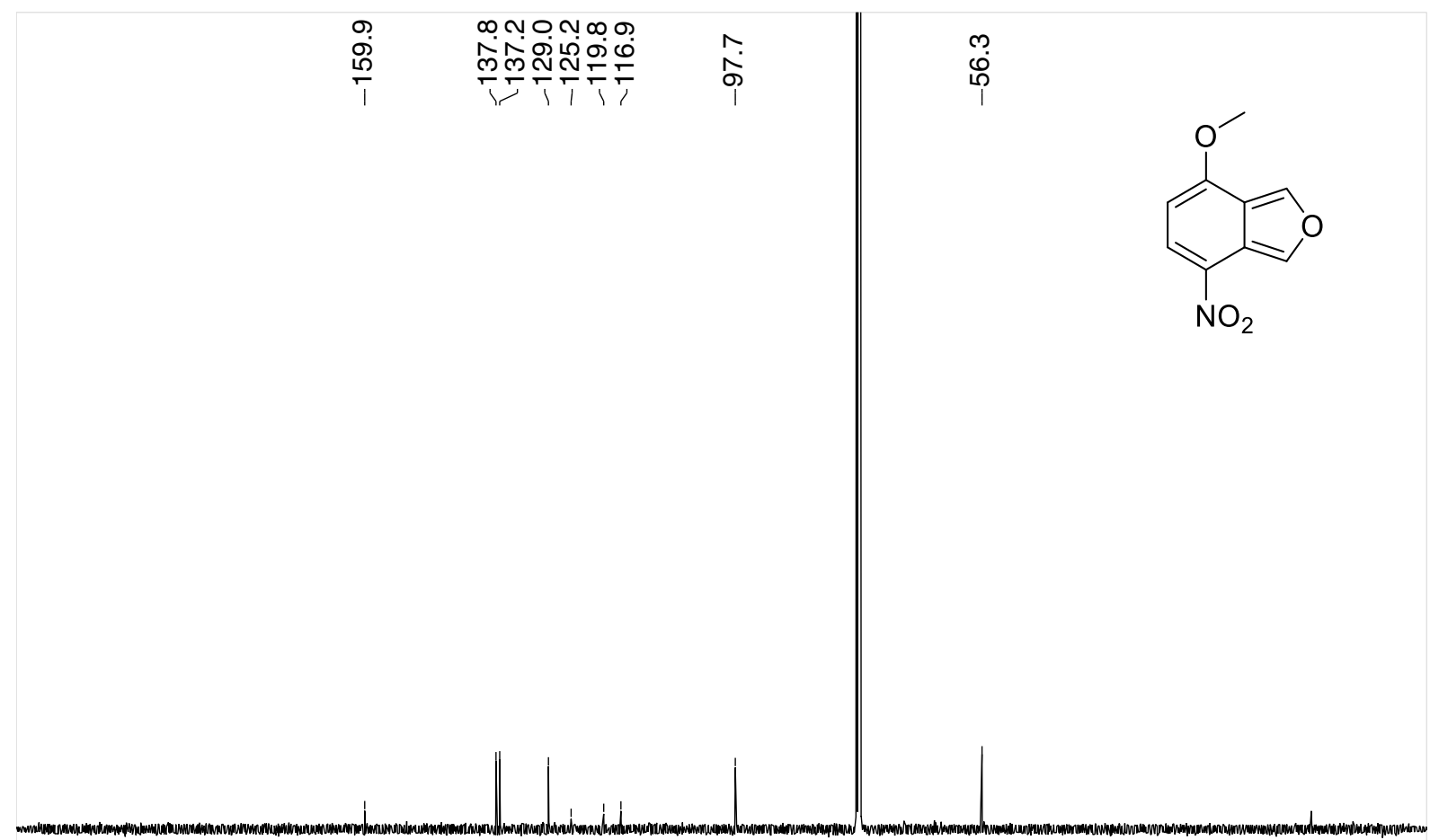

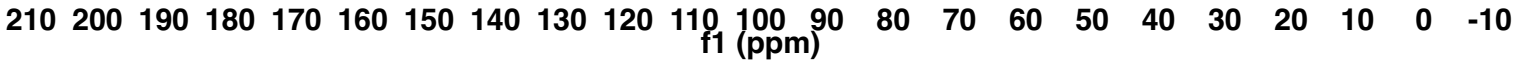

Figure $\mathrm{S} 14{ }^{13} \mathrm{C}\{1 \mathrm{H}\}$ NMR Compound 2-OMe in $\mathrm{CDCl}_{3}(100 \mathrm{MHz})$ 


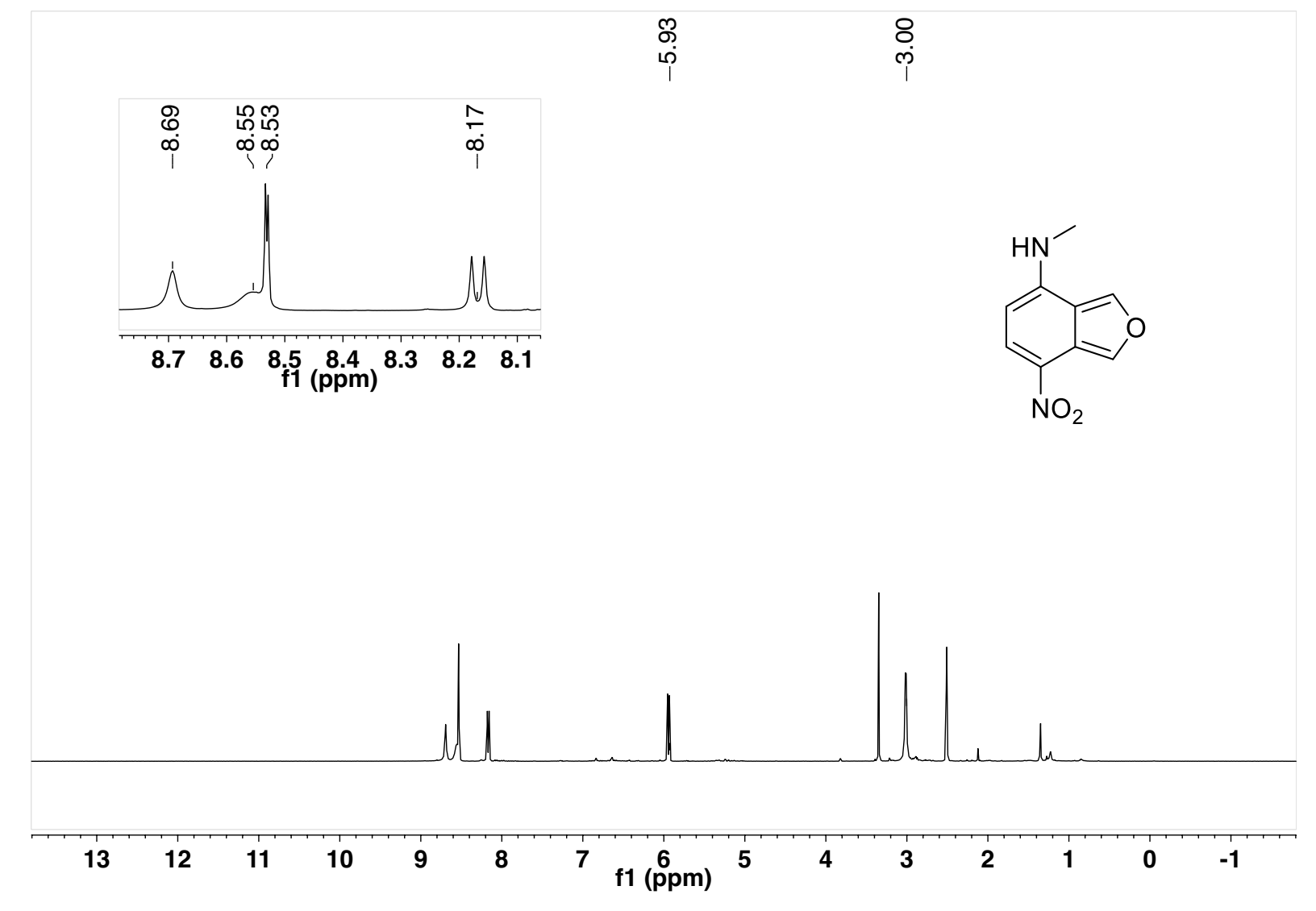

Figure S15 ${ }^{1} \mathrm{H}$ NMR Compound 2-NHMe in DMSO-d $(100 \mathrm{MHz})$ 


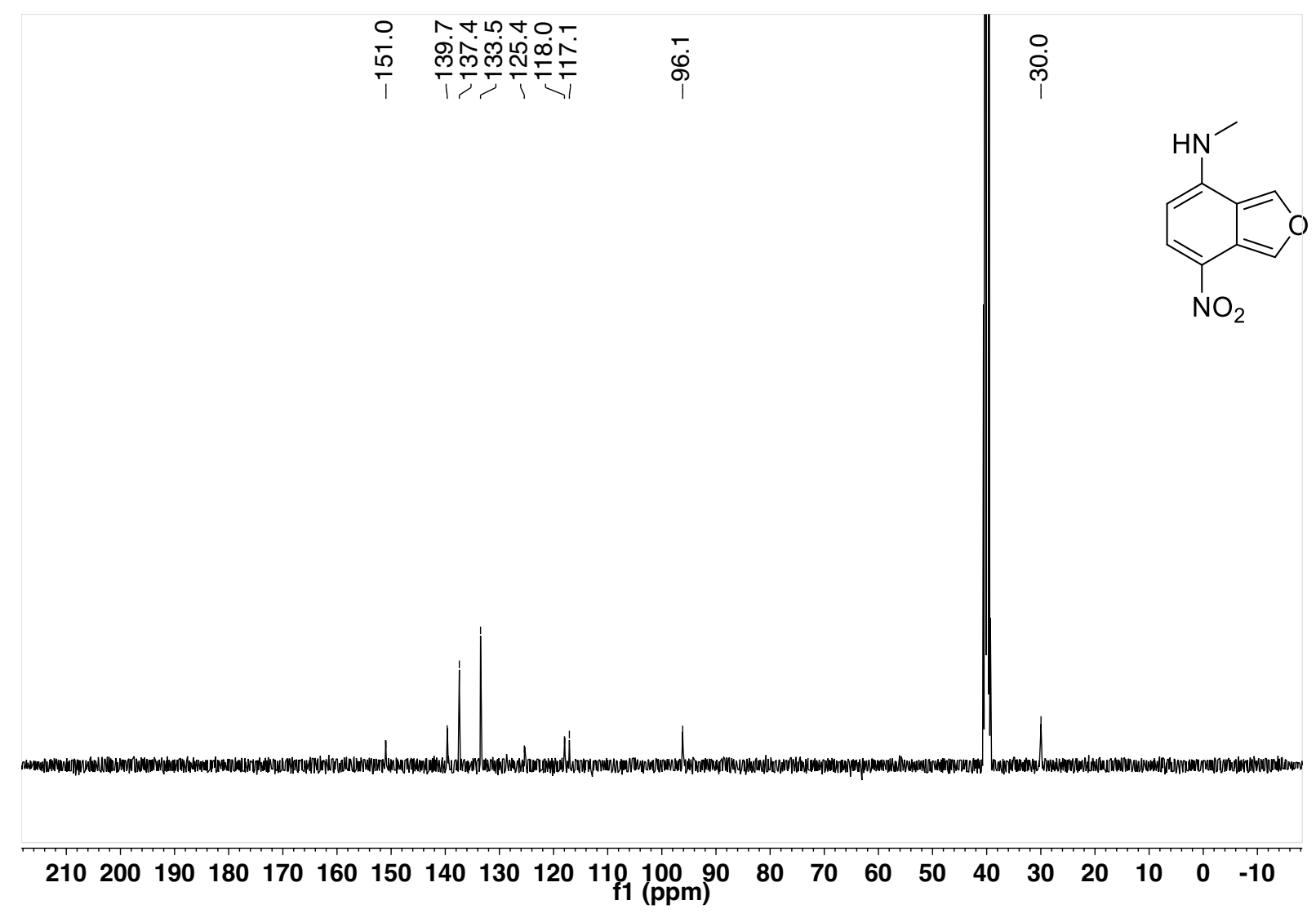

Figure $\mathrm{S} 16{ }^{13} \mathrm{C}\{1 \mathrm{H}\}$ NMR Compound 2-NHMe in DMSO-d $6(100 \mathrm{MHz})$ 


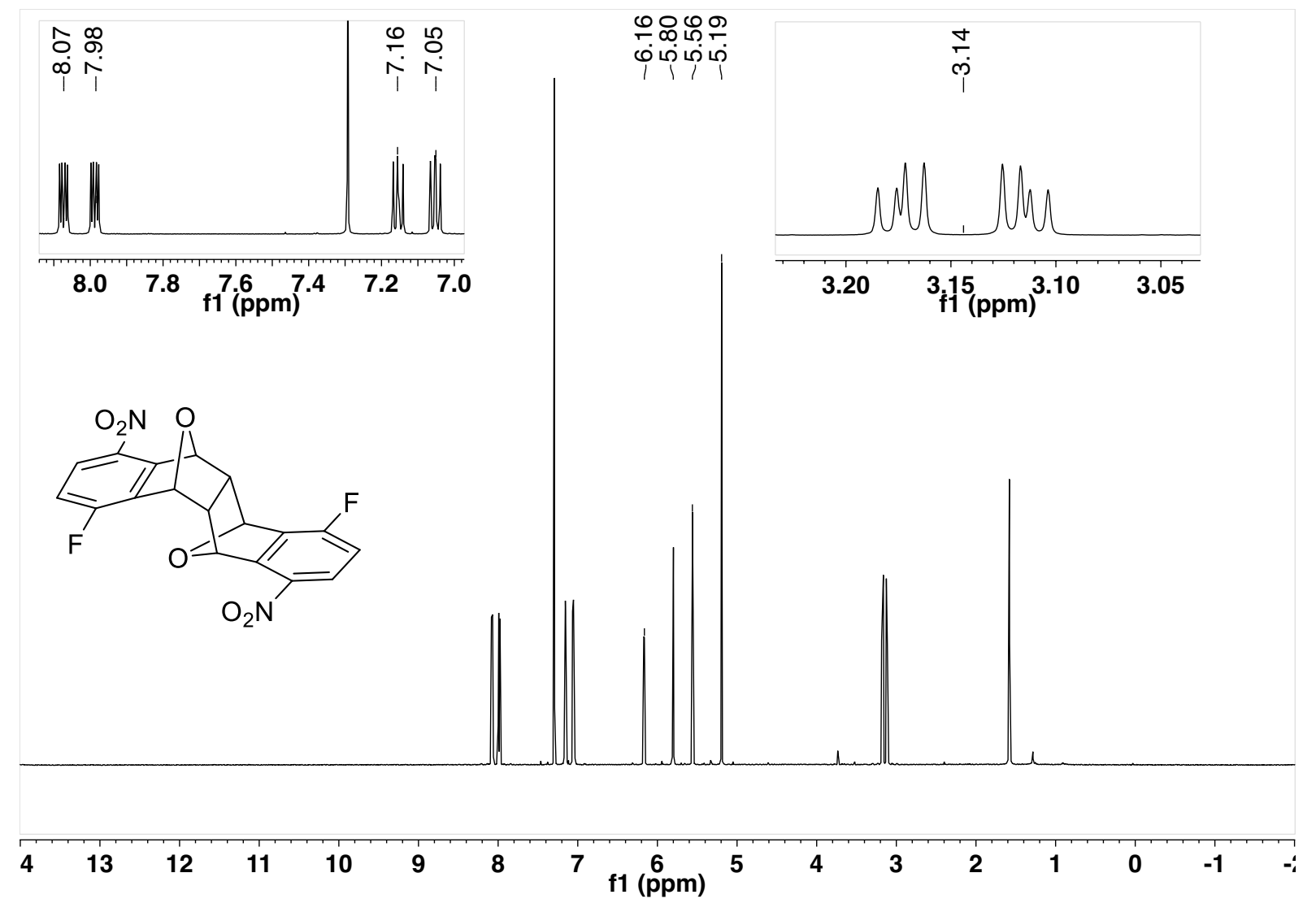

Figure $\mathrm{S} 17{ }^{1} \mathrm{H}$ NMR Compound 9 in $\mathrm{CDCl}_{3}(600 \mathrm{MHz})$ 


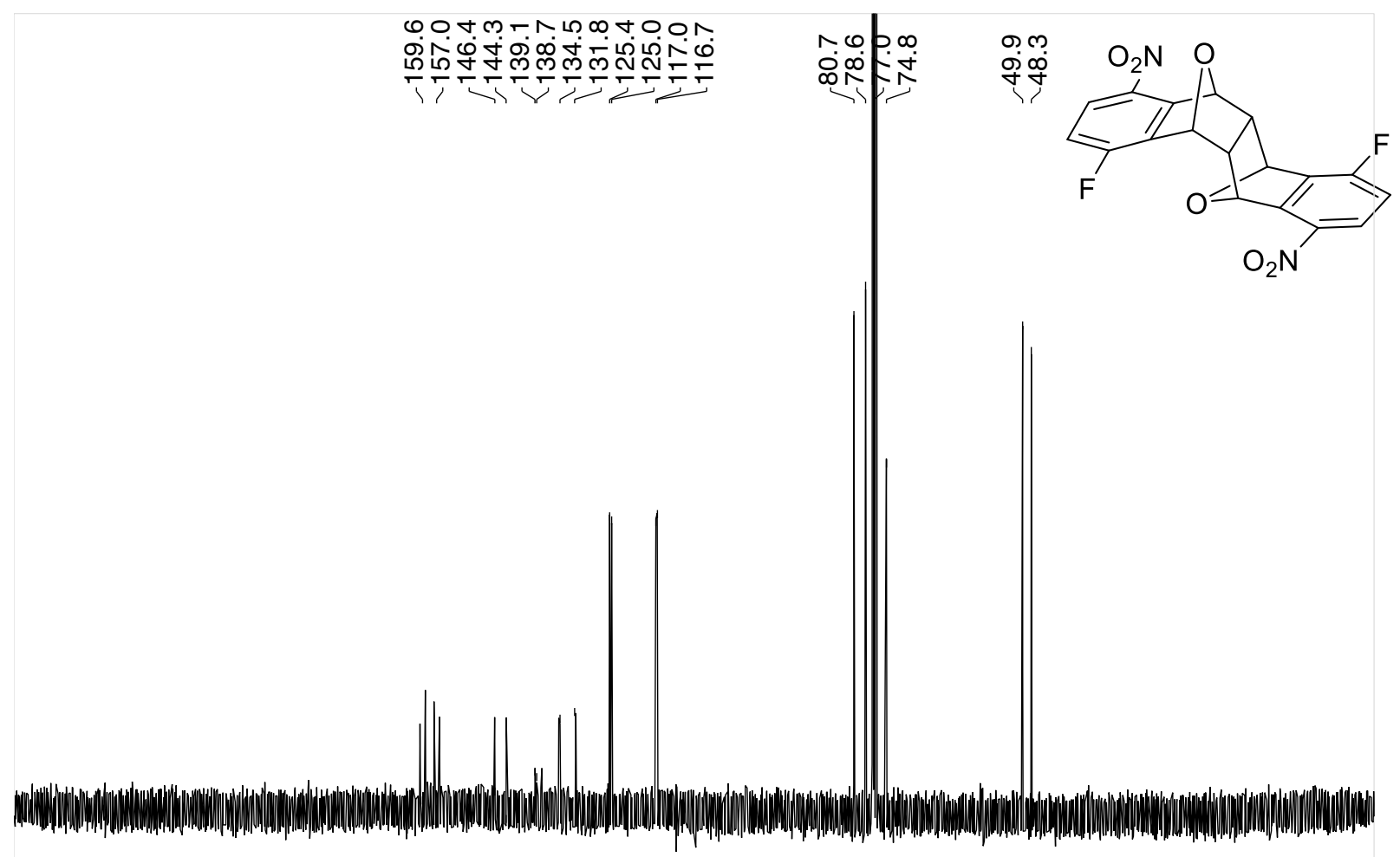

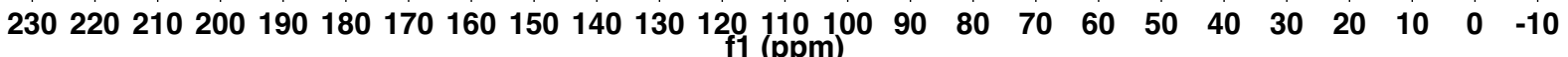

Figure $\mathrm{S} 18{ }^{13} \mathrm{C}\{1 \mathrm{H}\} \mathrm{NMR}$ Compound 9 in $\mathrm{CDCl}_{3}(100 \mathrm{MHz})$ 


\section{Spectral Data}

Table S1: Spectral Properties of 5

\begin{tabular}{|c|c|c|c|c|c|}
\hline & solvent & $\begin{array}{c}\text { abs } \lambda_{\max } \\
(\mathrm{nm})\end{array}$ & $\begin{array}{c}\text { em } \lambda_{\max } \\
530(\mathrm{~nm})\end{array}$ & $\log \varepsilon$ & $\phi_{\mathrm{F}}$ \\
\hline $\mathbf{5}$ & Toluene & 411 & 515 & 2.56 & 0.026 \\
\hline & Dioxane & 411 & 530 & 2.62 & 0.043 \\
\hline & $\mathrm{THF}$ & 410 & 528 & 2.64 & 0.059 \\
\hline & $\mathrm{CH}_{2} \mathrm{Cl}_{2}$ & 414 & 531 & 2.65 & 0.095 \\
\hline & $\mathrm{MeCN}$ & 413 & 559 & 2.55 & 0.19 \\
\hline & $\mathrm{MeOH}$ & 410 & 600 & 2.56 & 0.004 \\
\hline & $\mathrm{H}_{2} \mathrm{O}$ & 426 & $\mathrm{ND}$ & 2.44 & $\mathrm{ND}$ \\
\hline
\end{tabular}

Average of 2 measurements. Quantum yields determined relative to coumarin 153 in ethanol.

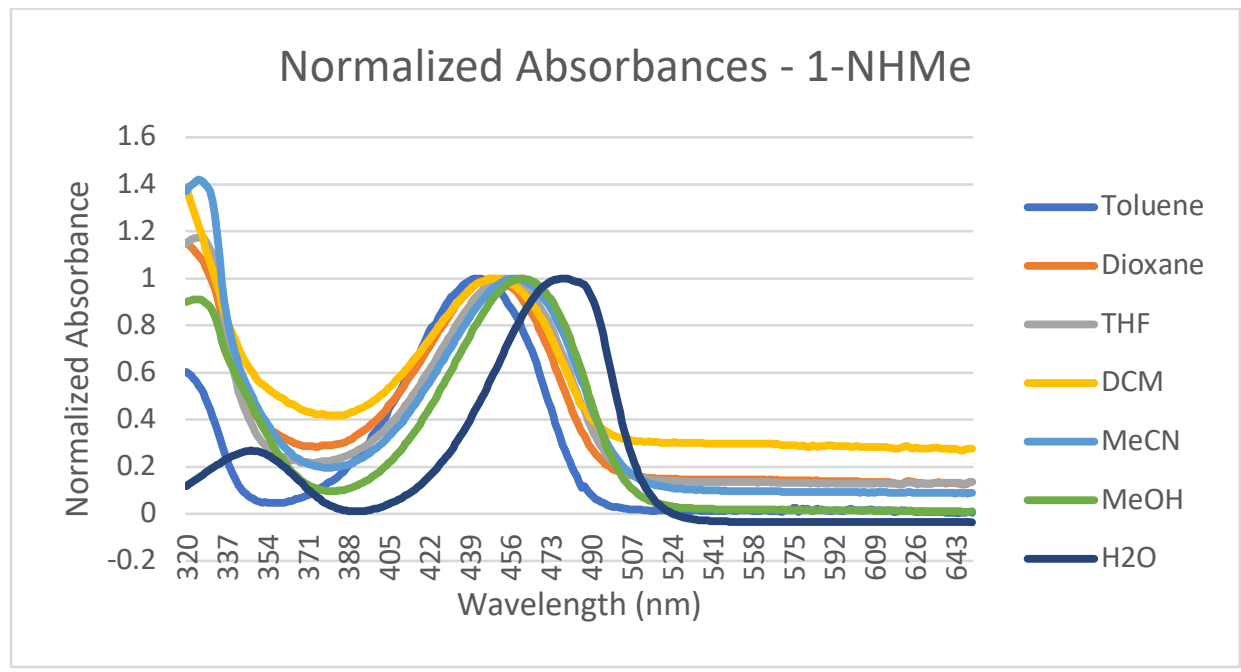

Figure S19: Normalized absorbance of 1-NHMe in various solvents

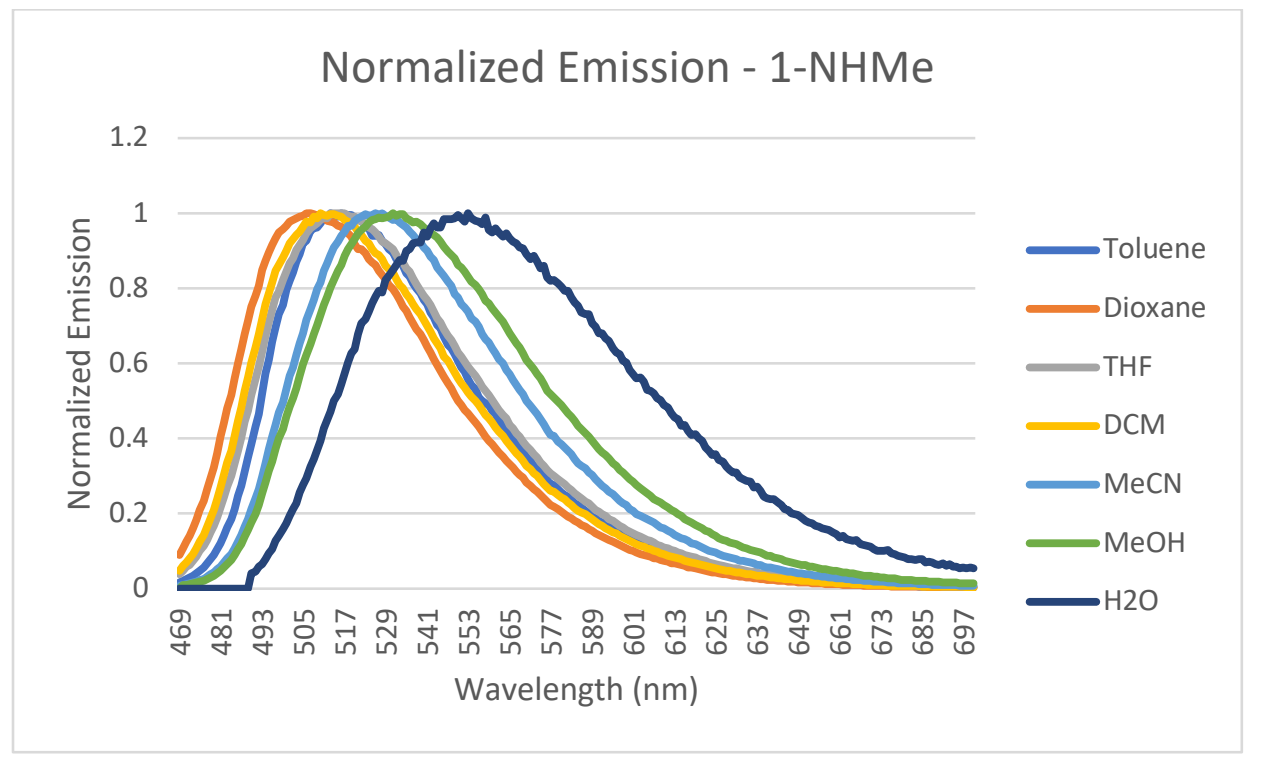

Figure S20: Normalized emission of 1-NHMe in various solvents 


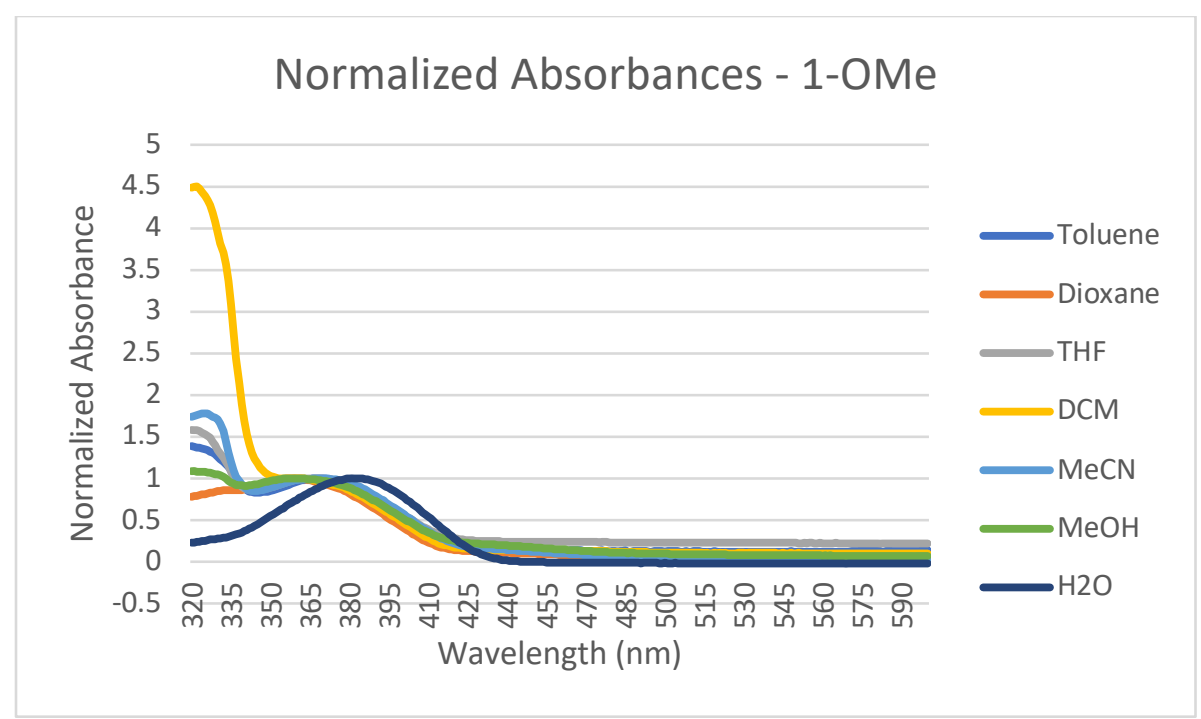

S21: Normalized absorbance of 1-OMe in various solvents

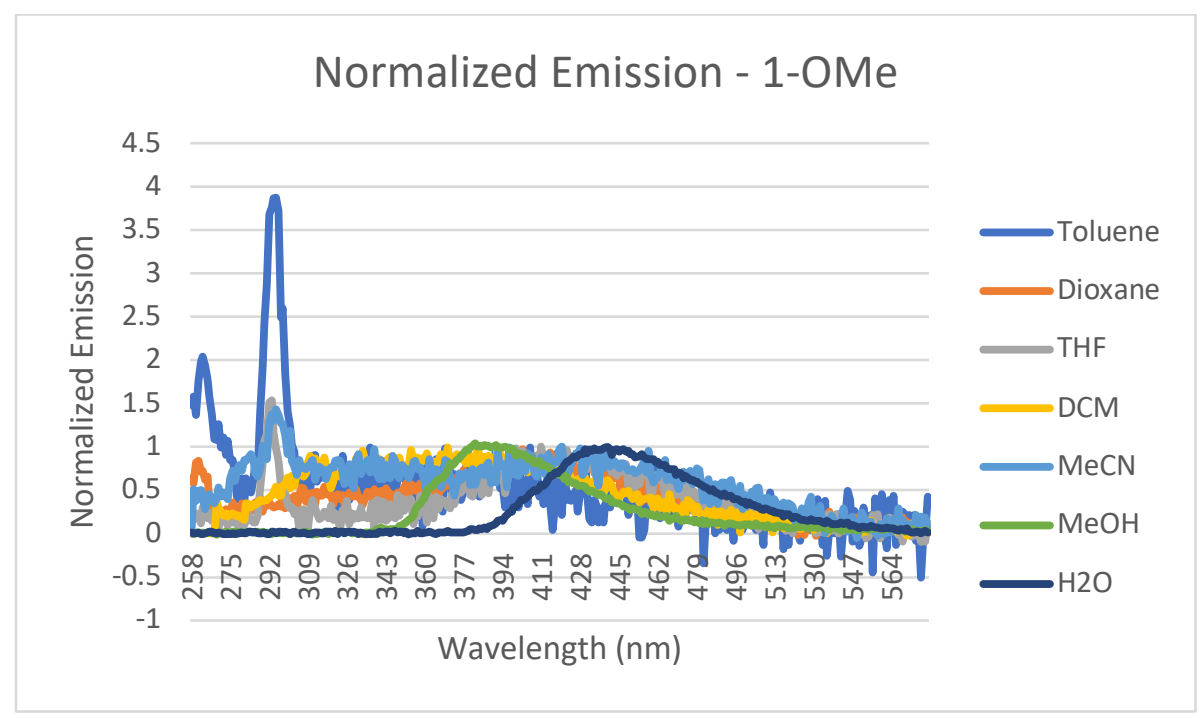

Figure S22: Normalized emission of 1-OMe in various solvents 


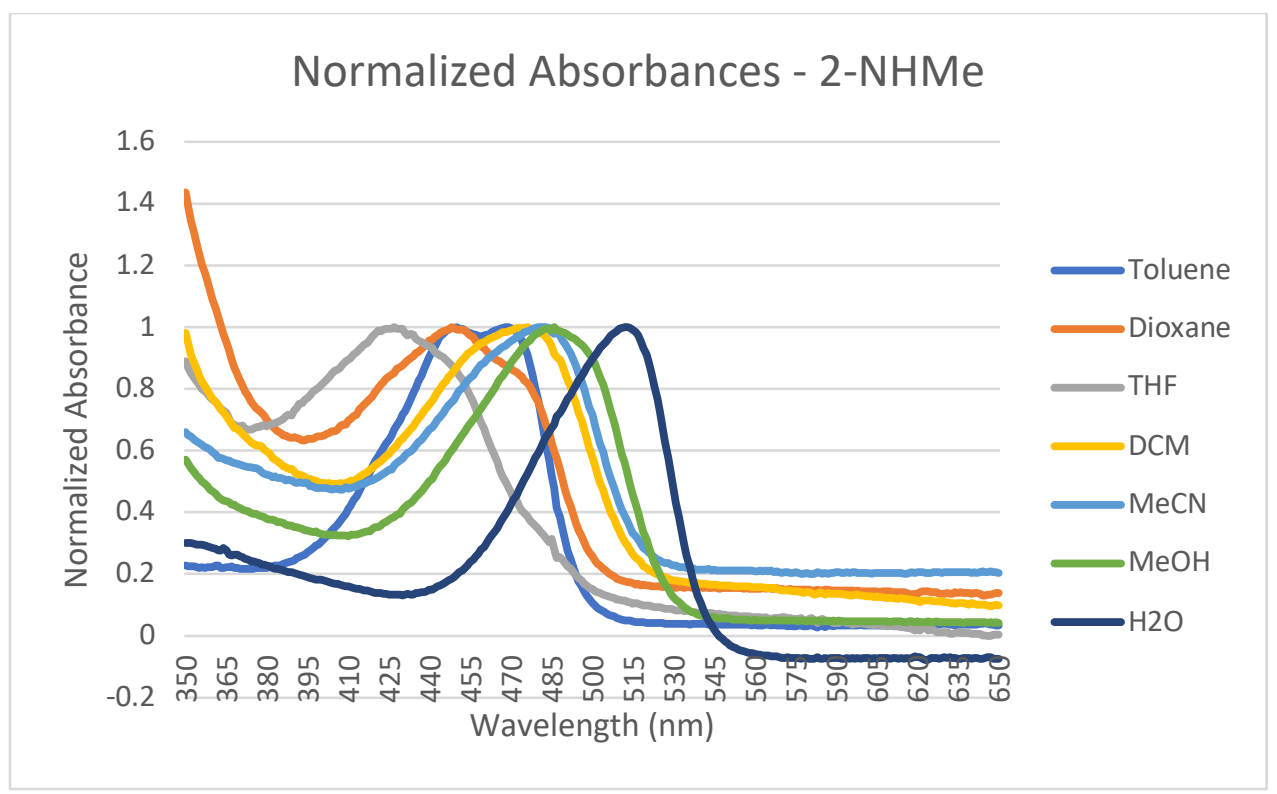

Figure S23: Normalized absorbance of 2-NHMe in various solvents

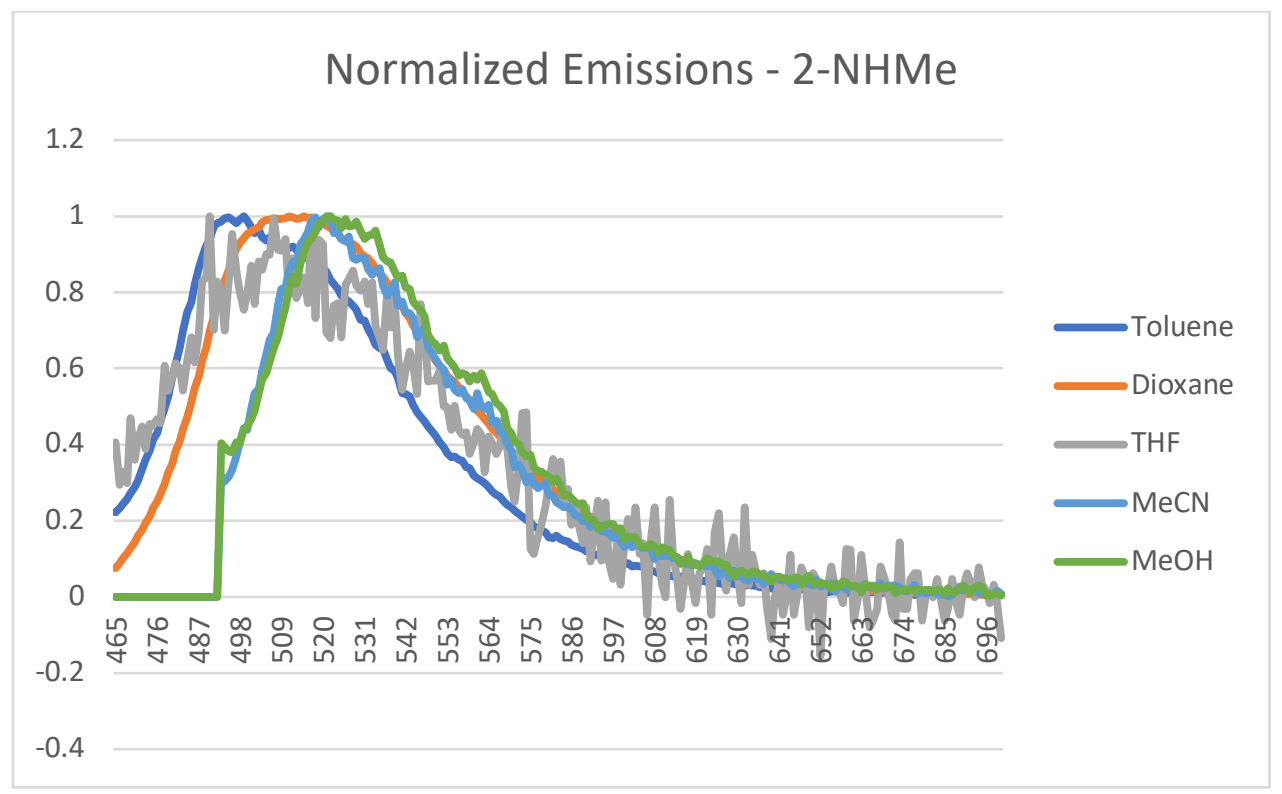

Figure S24: Normalized emission of 2-NHMe in various solvents. 


\section{Computational Details}

\section{Computational Methods}

All structures were optimized at the $6-31+g(d)$ basis set using the range-separated hybrid functional, $\omega B 97 X-D,{ }^{4}$ to account for the anticipated large amount charge transfer character. ${ }^{5}$ The excited state geometry optimizations were performed using TD- $\omega$ B97X-D with the same basis set. Vibrational analysis were done for all optimized structures at the same basis set and method and were verified to be a true minima by the absence of any imaginary frequencies. Excited state vibrational analysis were done numerically while ground state vibrational analysis were done analytically. Vertical excitation energies were calculated using the TD- $\omega$ B97X-D functional with the $6-311++g(2 d, p)$ basis set. All calculations were done using the Gaussian 09 suite of programs. ${ }^{6}$

\section{Computational Results}

A
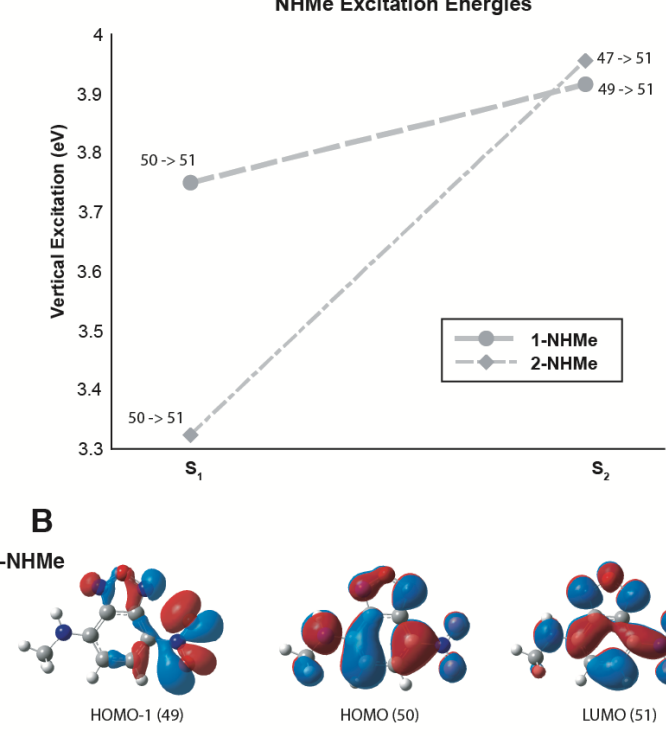

2-NHMe

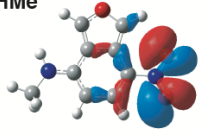

HOMO-3 (47)
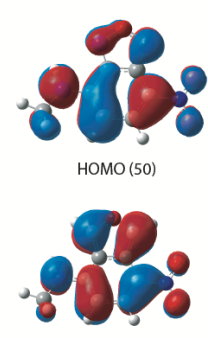

HOMO (50)
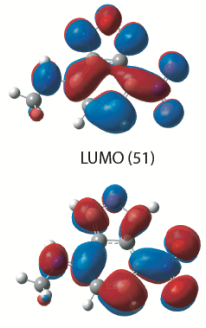

номо-3 (51)

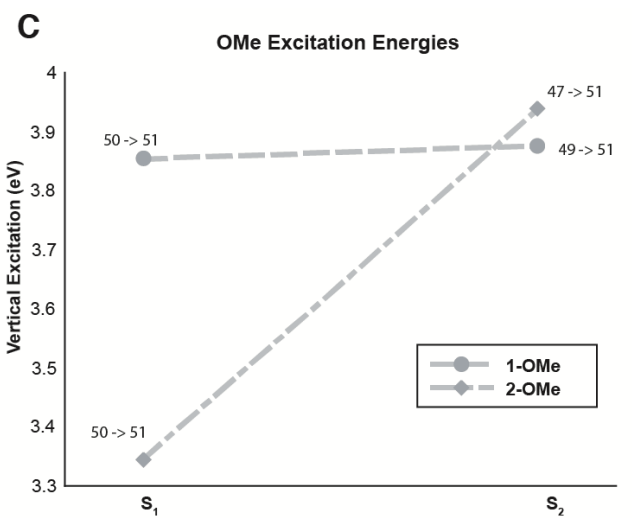

D
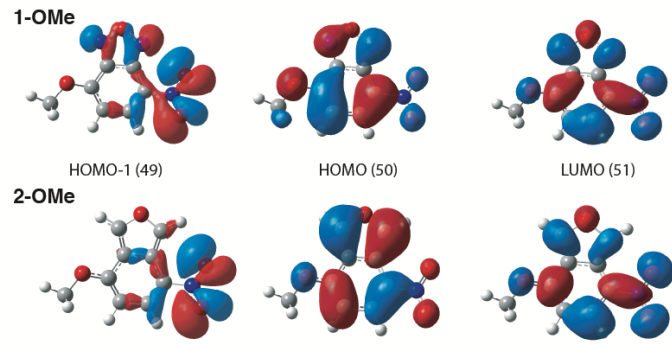

HOMO-3 (47)
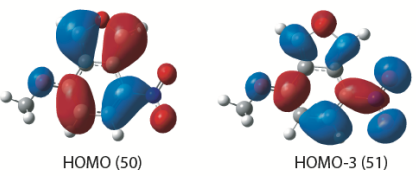

Figure S25. Calculated vertical excitation energies for (A) 1-NHMe, 2-NHMe, (B) 1-OMe, and 2-OMe and the molecular orbitals involved in each transition ( $B$ and $D)$. 


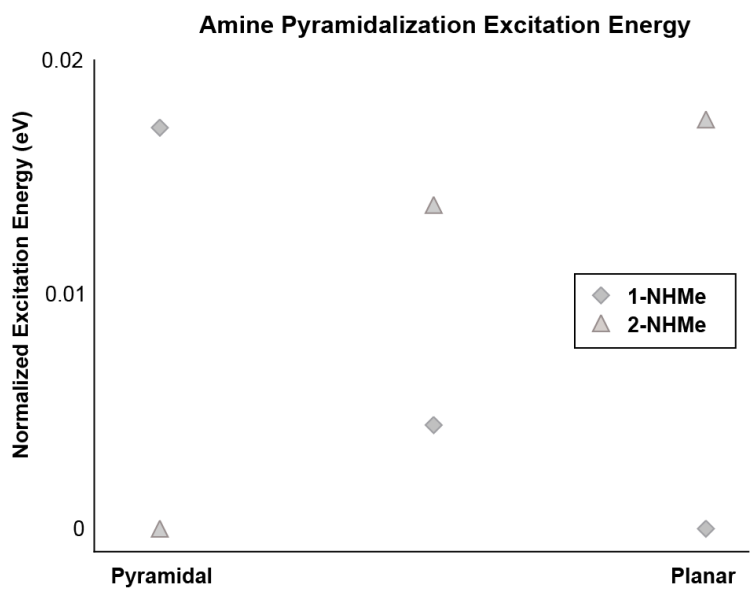

Figure S26. Calculated excitation energies for 1-NHMe and 2-NHMe along the amine pyramidalization coordinate. Reported energies are normalized to the lowest excitation energy for each molecule.

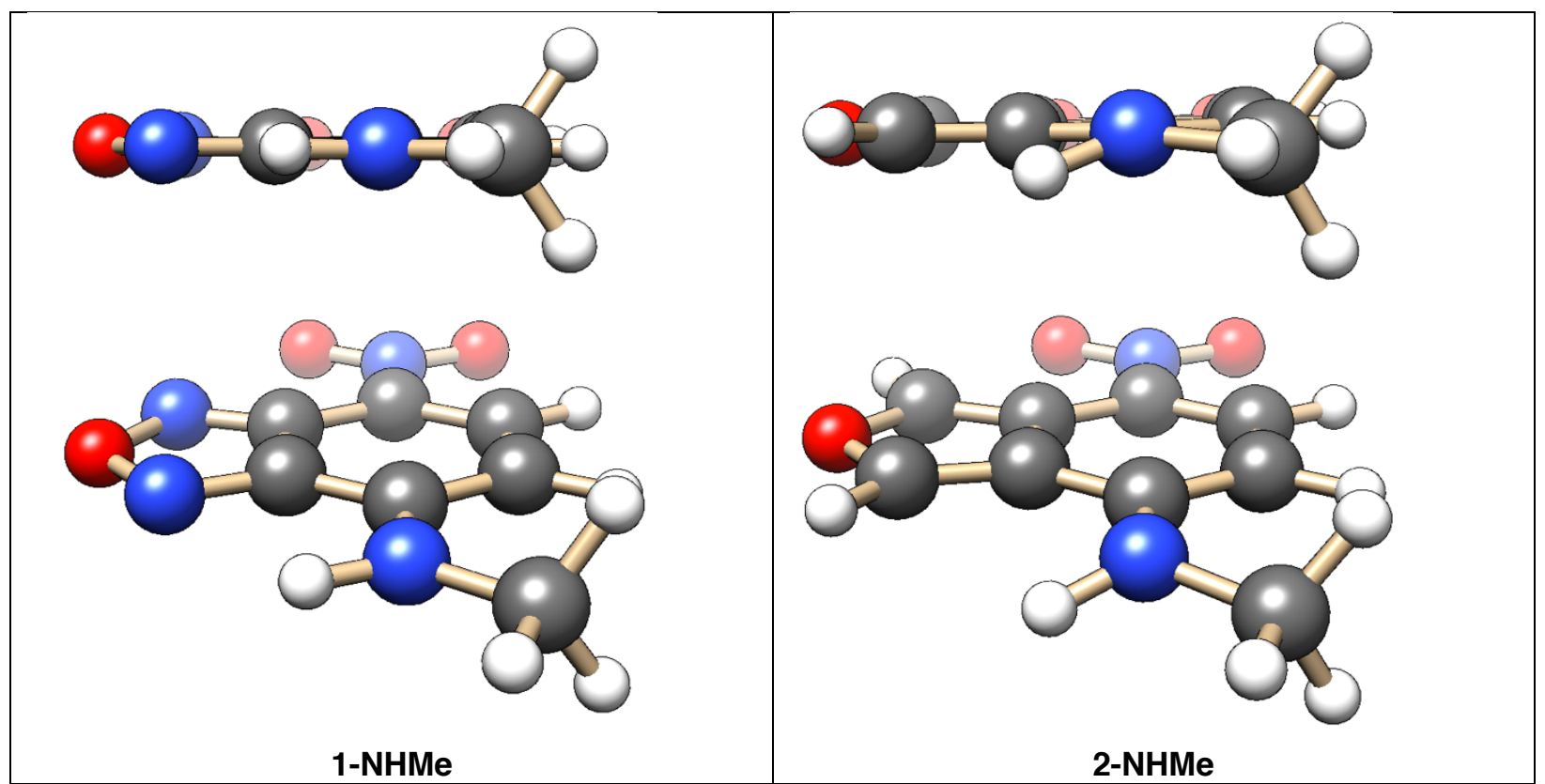

Figure S27. Excited-state structures of 1-NHMe and 2-NHMe

\section{Optimized Coordinates}

\begin{tabular}{lrrr} 
1-NHMe & \multicolumn{2}{c}{ Y } \\
C & -0.814275 & 0.819693 & 0.000078 \\
C & 0.611181 & 0.741693 & -0.000009 \\
C & 1.21979 & -0.552964 & 0.00001 \\
C & 0.392104 & -1.646357 & -0.000061 \\
C & -1.022505 & -1.56433 & -0.000103 \\
C & -1.673099 & -0.339975 & 0.000136 \\
H & 0.857378 & -2.626286 & -0.000126
\end{tabular}




$\begin{array}{lrrr}\mathrm{H} & -1.59041 & -2.487298 & -0.000291 \\ \mathrm{~N} & 2.65307 & -0.724096 & 0.000008 \\ \mathrm{O} & 3.098004 & -1.868355 & 0.000102 \\ \mathrm{O} & 3.336463 & 0.289614 & 0.000014 \\ \mathrm{O} & -0.01988 & 2.768452 & -0.000111 \\ \mathrm{C} & -3.957224 & -1.252847 & -0.000299 \\ \mathrm{H} & -4.965963 & -0.838621 & 0.002104 \\ \mathrm{H} & -3.838185 & -1.877432 & -0.893875 \\ \mathrm{H} & -3.835576 & -1.880811 & 0.890516 \\ \mathrm{~N} & -3.00889 & -0.161387 & 0.000601 \\ \mathrm{H} & -3.352493 & 0.787569 & -0.000358 \\ \mathrm{~N} & -1.172894 & 2.080325 & 0.000111 \\ \mathrm{~N} & 1.081958 & 1.973116 & -0.000224\end{array}$

$\begin{array}{ll}\text { Energy } & -714.6297871\end{array}$

\begin{tabular}{lrrr} 
1-OMe & \multicolumn{3}{c}{ Z } \\
C & -0.84017 & 0.830311 & -0.000154 \\
$\mathrm{C}$ & 0.585558 & 0.745088 & -0.000163 \\
$\mathrm{C}$ & 1.1952 & -0.550342 & -0.000056 \\
$\mathrm{C}$ & 0.380571 & -1.644639 & 0.00006 \\
$\mathrm{C}$ & -1.043186 & -1.556951 & 0.000136 \\
$\mathrm{C}$ & -1.678483 & -0.338785 & 0.000035 \\
$\mathrm{H}$ & 0.844969 & -2.624667 & 0.000142 \\
$\mathrm{H}$ & -1.608209 & -2.480971 & 0.000177 \\
$\mathrm{~N}$ & 2.638028 & -0.719814 & 0.000028 \\
$\mathrm{O}$ & 3.079574 & -1.863217 & 0.000047 \\
$\mathrm{O}$ & 3.316389 & 0.294217 & -0.000138 \\
$\mathrm{O}$ & -0.028336 & 2.769258 & 0.000768 \\
$\mathrm{C}$ & -3.863451 & -1.236065 & 0.000092 \\
$\mathrm{H}$ & -4.869576 & -0.819718 & 0.000351 \\
$\mathrm{H}$ & -3.711826 & -1.843487 & -0.89893 \\
$\mathrm{H}$ & -3.711368 & -1.843473 & 0.899046 \\
$\mathrm{~N}$ & -1.189795 & 2.096812 & -0.000643 \\
$\mathrm{~N}$ & 1.067602 & 1.971725 & -0.000093 \\
$\mathrm{O}$ & -2.98901 & -0.115314 & -0.000117
\end{tabular}




\begin{tabular}{lrrr} 
2-NHMe & \multicolumn{3}{c}{ Z } \\
C & X & \multicolumn{3}{c}{ Y } \\
C & -0.62008 & 0.706868 & 0.000793 \\
$\mathrm{C}$ & -1.184555 & -0.60952 & -0.002117 \\
$\mathrm{C}$ & -0.351831 & -1.696409 & -0.011606 \\
$\mathrm{C}$ & 1.057633 & -1.572641 & -0.01596 \\
$\mathrm{C}$ & 1.672334 & -0.329335 & -0.014932 \\
$\mathrm{C}$ & 1.079286 & 2.179903 & -0.008799 \\
$\mathrm{C}$ & -1.108172 & 1.985628 & 0.007265 \\
$\mathrm{H}$ & -0.797073 & -2.685337 & -0.013138 \\
$\mathrm{H}$ & 1.65248 & -2.478627 & -0.018593 \\
$\mathrm{H}$ & 1.978704 & 2.776586 & -0.020572 \\
$\mathrm{H}$ & -2.097998 & 2.407147 & 0.015474 \\
$\mathrm{~N}$ & -2.608713 & -0.79336 & 0.004657 \\
$\mathrm{O}$ & -3.063296 & -1.934364 & -0.000471 \\
$\mathrm{O}$ & -3.30539 & 0.22116 & 0.015274 \\
$\mathrm{O}$ & -0.075615 & 2.862789 & 0.001477 \\
$\mathrm{C}$ & 3.956039 & -1.266982 & 0.033582 \\
$\mathrm{H}$ & 4.970313 & -0.865326 & 0.012209 \\
$\mathrm{H}$ & 3.828561 & -1.851317 & 0.954206 \\
$\mathrm{H}$ & 3.83275 & -1.934556 & -0.827075 \\
$\mathrm{~N}$ & 3.020845 & -0.166704 & -0.030701 \\
$\mathrm{H}$ & 3.386379 & 0.765423 & 0.071315
\end{tabular}

Energy $\quad-682.6222554$

\begin{tabular}{lrrr} 
2-OMe & \multicolumn{2}{c}{ Y } \\
C & -0.844103 & 0.813002 & 0.000079 \\
$\mathrm{C}$ & 0.595851 & 0.715321 & 0.000063 \\
$\mathrm{C}$ & 1.175231 & -0.594549 & -0.000029 \\
$\mathrm{C}$ & 0.367965 & -1.695104 & -0.00012 \\
$\mathrm{C}$ & -1.05297 & -1.593921 & -0.000113 \\
$\mathrm{C}$ & -1.66348 & -0.361777 & 0.000006 \\
$\mathrm{H}$ & 0.829259 & -2.676416 & -0.000224 \\
$\mathrm{H}$ & -1.630389 & -2.510267 & -0.000228 \\
$\mathrm{~N}$ & 2.611059 & -0.758558 & -0.000051 \\
$\mathrm{O}$ & 3.078309 & -1.892019 & -0.000171 \\
$\mathrm{O}$ & 3.288192 & 0.265798 & 0.000051
\end{tabular}




$\begin{array}{lrrr}\mathrm{O} & 0.00051 & 2.855307 & 0.000337 \\ \mathrm{C} & -3.861454 & -1.249486 & -0.000096 \\ \mathrm{H} & -4.868048 & -0.832698 & -0.000029 \\ \mathrm{H} & -3.713376 & -1.859773 & -0.898084 \\ \mathrm{H} & -3.713327 & -1.859941 & 0.897771 \\ \mathrm{O} & -2.984568 & -0.134112 & -0.000016 \\ \mathrm{C} & -1.140837 & 2.149681 & 0.000053 \\ \mathrm{C} & 1.055054 & 2.005124 & 0.000058 \\ \mathrm{H} & -2.062646 & 2.708279 & 0.000066 \\ \mathrm{H} & 2.034047 & 2.451197 & 0.00007\end{array}$

Energy

$-702.4735851$

\section{1-NHMe s1}

\begin{tabular}{lrrr} 
& \multicolumn{3}{c}{$\mathrm{Y}$} \\
$\mathrm{C}$ & $\mathrm{X}$ & \multicolumn{2}{r}{$\mathrm{Z}$} \\
$\mathrm{C}$ & 0.811901 & 0.829534 & 0.00004 \\
$\mathrm{C}$ & 1.19985 & -0.549159 & -0.000204 \\
$\mathrm{C}$ & 0.383253 & -1.708882 & -0.000305 \\
$\mathrm{C}$ & -0.993995 & -1.606747 & -0.00018 \\
$\mathrm{C}$ & -1.640258 & -0.324202 & 0.000092 \\
$\mathrm{H}$ & 0.872276 & -2.674453 & -0.000446 \\
$\mathrm{H}$ & -1.595732 & -2.508914 & -0.000295 \\
$\mathrm{~N}$ & 2.627872 & -0.718004 & -0.000117 \\
$\mathrm{O}$ & 3.062218 & -1.87467 & 0.000517 \\
$\mathrm{O}$ & 3.317545 & 0.293903 & 0.000005 \\
$\mathrm{O}$ & -0.01041 & 2.805219 & -0.000016 \\
$\mathrm{C}$ & -3.94773 & -1.24018 & -0.000136 \\
$\mathrm{H}$ & -4.943984 & -0.798047 & 0.002621 \\
$\mathrm{H}$ & -3.845388 & -1.865353 & -0.894762 \\
$\mathrm{H}$ & -3.842276 & -1.869081 & 0.891471 \\
$\mathrm{~N}$ & -2.973019 & -0.168927 & 0.000477 \\
$\mathrm{H}$ & -3.305166 & 0.78883 & 0.000322 \\
$\mathrm{~N}$ & -1.200136 & 2.089105 & 0.000136 \\
$\mathrm{~N}$ & 1.107173 & 1.970845 & -0.000197 \\
& & & \\
& & & -714.507981
\end{tabular}

2-NHMe_s1

$X$

Y

Z 


$\begin{array}{lrrr}\mathrm{C} & 0.806453 & 0.837435 & -0.015384 \\ \mathrm{C} & -0.624488 & 0.670315 & 0.009715 \\ \mathrm{C} & -1.179765 & -0.625793 & -0.000565 \\ \mathrm{C} & -0.30232 & -1.743521 & -0.034761 \\ \mathrm{C} & 1.076012 & -1.568084 & -0.044942 \\ \mathrm{C} & 1.685338 & -0.290376 & -0.02537 \\ \mathrm{C} & 1.014944 & 2.194783 & -0.032446 \\ \mathrm{C} & -1.157506 & 1.946913 & 0.017491 \\ \mathrm{H} & -0.739416 & -2.733974 & -0.046012 \\ \mathrm{H} & 1.703724 & -2.452686 & -0.059477 \\ \mathrm{H} & 1.899227 & 2.813515 & -0.077631 \\ \mathrm{H} & -2.16913 & 2.315989 & 0.036061 \\ \mathrm{~N} & -2.57846 & -0.804966 & 0.012859 \\ \mathrm{O} & -3.01227 & -1.973484 & -0.000295 \\ \mathrm{O} & -3.305097 & 0.212509 & 0.038015 \\ \mathrm{O} & -0.163045 & 2.87272 & -0.006682 \\ \mathrm{C} & 3.979139 & -1.21048 & 0.065887 \\ \mathrm{H} & 4.993598 & -0.809233 & 0.031654 \\ \mathrm{H} & 3.850414 & -1.770742 & 1.003631 \\ \mathrm{H} & 3.861485 & -1.901789 & -0.775069 \\ \mathrm{~N} & 3.045975 & -0.110017 & -0.052085 \\ \mathrm{H} & 3.38395 & 0.782675 & 0.275379\end{array}$

$-682.5048636$

\section{REFERENCES}

(1) Mjalli, A. M. M.; Jones, D.; Gohimmukkula, D. R.; Huang, G.; Zhu, J.; Rao, M.; Andrews, R. C.; Ren, T. Benzazole Derivatives, Compositions, and Methods of Use as B-Secretase Inhibitors. WO2006099379, 2006.

(2) Bencivenni, G.; Cesari, R.; Nanni, D.; El Mkami, H.; Walton, J. C., Epr and Endor Spectroscopic Study of the Reactions of Aromatic Azides with Gallium Trichloride. Org Biomol Chem 2010, 8, 5097-104. (3) Chan, S. H.; Yick, C. Y.; Wong, H. N. C., 5,6-Bis(Trimethylsilyl)Benzo[C]Furan: An Isolable Versatile Building Block for Linear Polycyclic Aromatic Compounds. Tetrahedron 2002, 58, 9413-9422. (4) Chai, J.-D.; Head-Gordon, M., Long-Range Corrected Hybrid Density Functionals with Damped Atom-Atom Dispersion Corrections. Physical Chemistry Chemical Physics 2008, 10, 6615-6620.

(5) Laurent, A. D.; Jacquemin, D., Td-Dft Benchmarks: A Review. International Journal of Quantum Chemistry 2013, 113, 2019-2039.

(6) M. J. Frisch, G. W. Trucks, H. B. Schlegel, G. E. Scuseria, M. A. Robb, J. R. Cheeseman, G. Scalmani, V. Barone, B. Mennucci, G. A. Petersson, H. Nakatsuji, M. Caricato, X. Li, P. H. Hratchian, A. F. Izmaylov, J. Bloino, G. Zheng, J. L. Sonnenberg, M. Hada, M. Ehara, K. Toyota, R. Fukuda, J. Hasegawa, M. Ishida, T. Nakajima, Y. Honda, O. Kitao, H. Nakai, T. Vreven, J. A. Montgomery Jr J. E. Peralta, F. Ogliaro, M. Bearpark, J. Heyd, E. Brothers, K. N. Kudin, V. N. Staroverov, R. Kobayashi, J. Normand, K. Raghavachari, A. Rendell, J. C. Burant, S. S. Iyengar, J. Tomasi, M. Cossi, N. Rega, J. M. 
Millam, M. Klene, J. E. Knox, J. B. Cross, V. Bakken, C. Adamo, J. Jaramillo, R. Gomperts, R. E. Stratmann, O. Yazyev, A. J. Austin, R. Cammi, C. Pomelli, J. W. Ochterski, R. L. Martin, K. Morokuma, V. G. Zakrzewski, G. A. Voth, P. Salvador, J. J. Dannenberg, S. Dapprich, A. D. Daniels, O. Farkas, J. B. Foresman, J. V. Ortiz, J. Cioslowski and D. J. Fox, Gaussian 09, Gaussian, Inc., Wallingford, CT, 2009. 